\title{
Transverse Momentum Distributions of Final-State Particles Produced in Soft Excitation Process in High Energy Collisions
}

\author{
Fu-Hu Liu, Ya-Hui Chen, Hua-Rong Wei, and Bao-Chun Li \\ Institute of Theoretical Physics, Shanxi University, Taiyuan, Shanxi 030006, China \\ Correspondence should be addressed to Fu-Hu Liu; fuhuliu@163.com
}

Received 17 July 2013; Revised 5 September 2013; Accepted 20 September 2013

Academic Editor: Gonang Xie

Copyright ( $) 2013 \mathrm{Fu}-\mathrm{Hu}$ Liu et al. This is an open access article distributed under the Creative Commons Attribution License, which permits unrestricted use, distribution, and reproduction in any medium, provided the original work is properly cited.

\begin{abstract}
Transverse momentum distributions of final-state particles produced in soft process in proton-proton ( $p p$ ) and nucleus-nucleus $(A A)$ collisions at Relativistic Heavy Ion Collider (RHIC) and Large Hadron Collider (LHC) energies are studied by using a multisource thermal model. Each source in the model is treated as a relativistic and quantum ideal gas. Because the quantum effect can be neglected in investigation on the transverse momentum distribution in high energy collisions, we consider only the relativistic effect. The concerned distribution is finally described by the Boltzmann or two-component Boltzmann distribution. Our modeling results are in agreement with available experimental data.
\end{abstract}

\section{Introduction}

High energy collisions are an important research topic in particle and nuclear physics. The Relativistic Heavy Ion Collider (RHIC) at Brookhaven National Laboratory (BNL) did firstly collider experiments on heavy ions [1], and the center-of-mass energy per nucleon pair $\left(\sqrt{s_{N N}}\right)$ at the RHIC reached highly $200 \mathrm{GeV}$ [2]. The Large Hadron Collider (LHC) at European Laboratory for Particle Physics (CERN) renovated value of $\sqrt{s_{N N}}$ to $\mathrm{TeV}$ region [3]. It seems that a new state of matter, namely, Quark-Gluon Plasma (QGP), is possibly formed in heavy ion collisions at RHIC and LHC energies due to high temperature and density $[4,5]$. At initial stage of high energy collisions, another possible new state of matter, namely, color glass condensate (CGC), is caused by strong color fields in the low- $x$ gluon realm $[6,7]$, where $x$ denotes the ratio of quark or gluon momentum to hadron one. A CGC is in fact a region of the nuclear wave function at low- $x$ and $Q^{2}$ and exists already before the collisions, where $Q^{2}$ denotes the square momentum of virtual photon. On the other hand, the CGC may not be a new state, but more like a model or calculation for initial state hadron behavior. One cannot measure the QGP and CGC directly. However, one can measure final-state particle spectra at freeze-out to extract thermal and other characteristics of interacting system and give a judgment on formation and property of the new matters.

The final-state particle spectra include rapidity $y$ (or pseudorapidity $\eta$ ) $[8,9]$, transverse momentum $p_{T}$ (or transverse mass) $[10,11]$, transverse energy $[12,13]$, and other distributions [14]. It is known that $y$ and $p_{T}$ distributions reflect, respectively, the degrees of longitudinal extension and transverse excitation of interacting system. Especially, for transverse excitation, soft excitation and hard scattering processes can affect, respectively, distributions in low- and high- $p_{T}$ ranges. The soft and hard processes correspond to different physics mechanisms and distribution laws [15]. In low energy collisions, the soft process is main process, and the hard process can be neglected due to almost zero contribution. In high energy collisions, although the hard process cannot be neglected, the soft process is still main process.

To understand the transverse excitation, we need firstly to study the soft excitation process. A lot of models have been introduced to describe the soft process, although some of them can be used to describe the hard process too $[16,17]$. Among the models, the multisource thermal model proposed by us is a very simple one and can be used to describe $p_{T}$ spectra in both the soft and hard processes if source's contribution is given by an Erlang distribution [18]. Finally, 


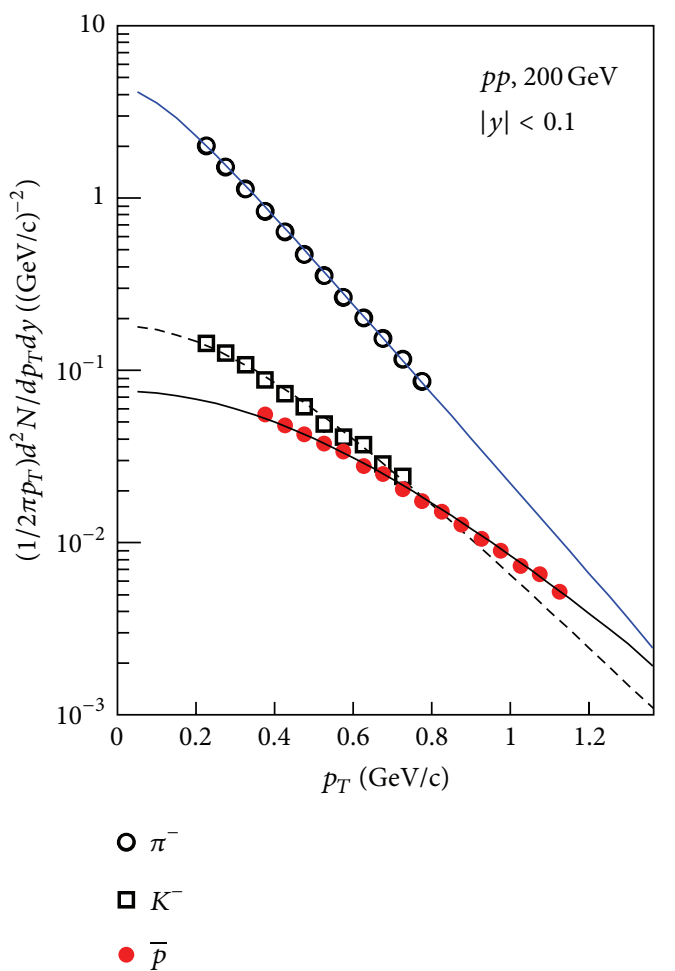

(a)

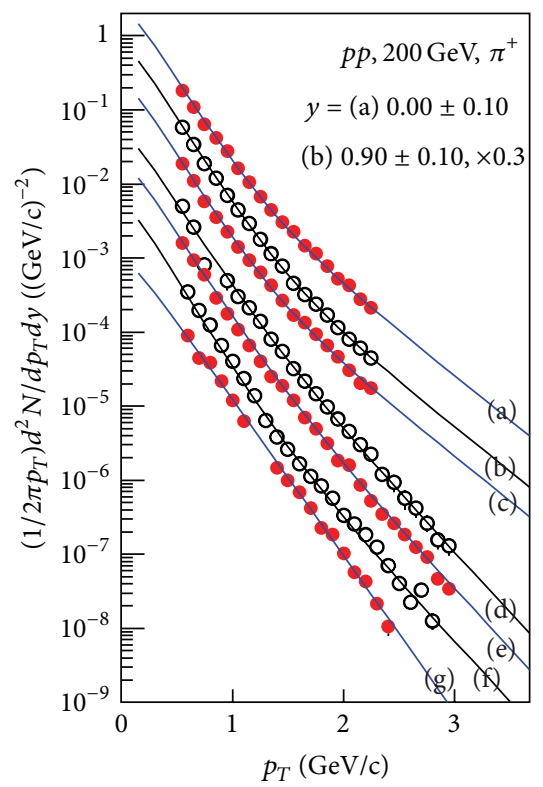

(c)

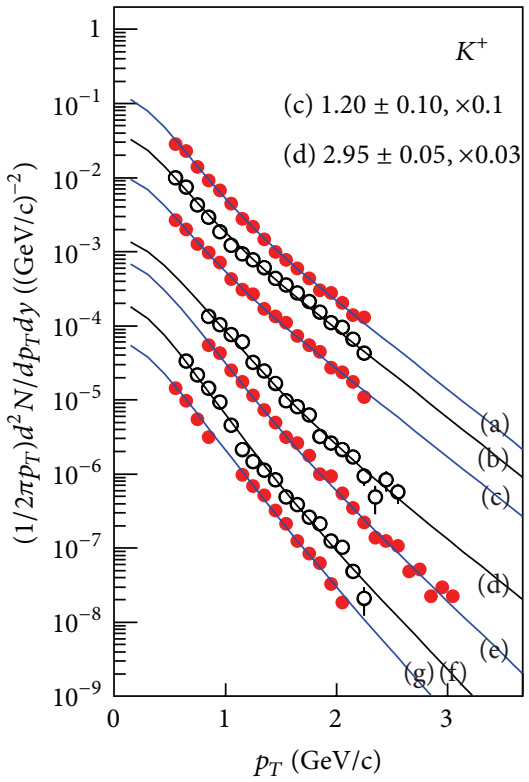

(d)

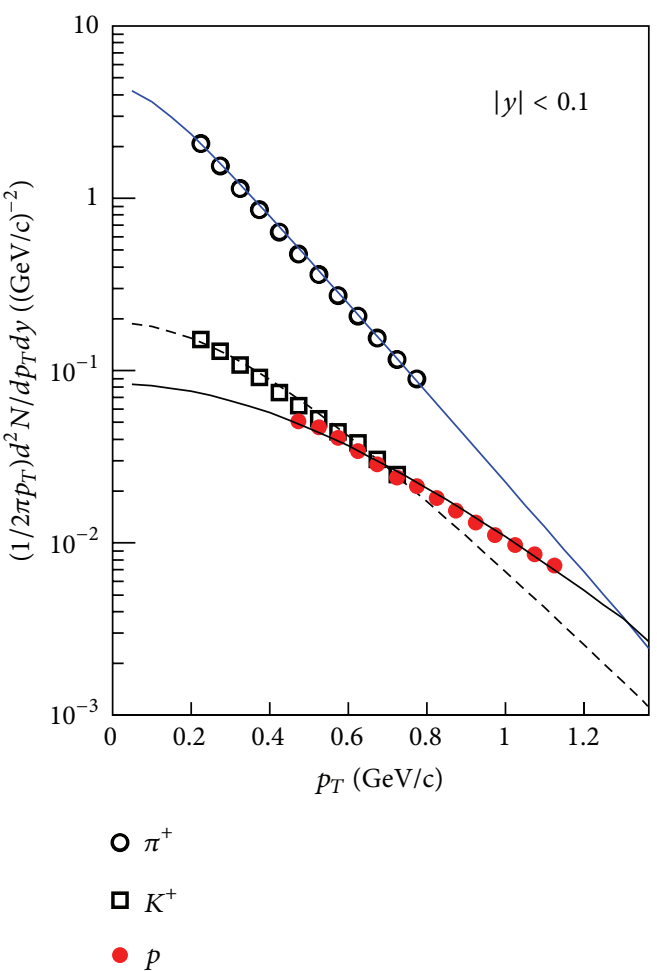

(b)

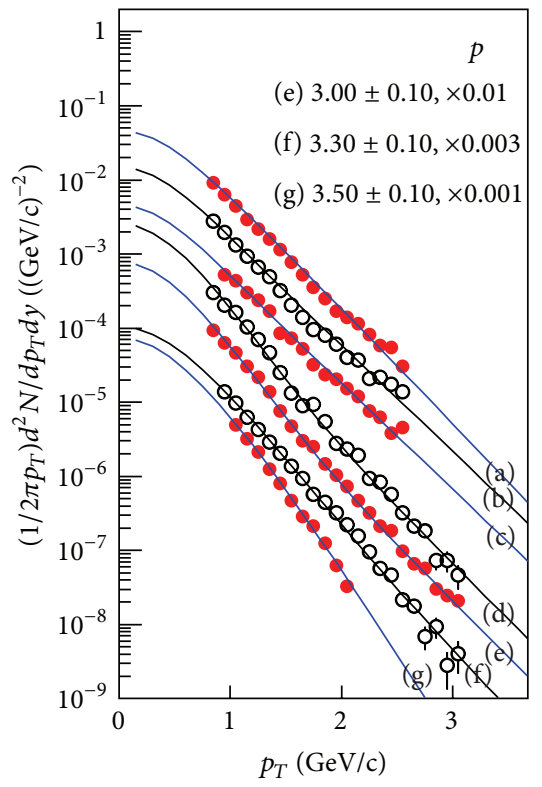

(e)

FIgURE 1: The transverse momentum distributions of (a) $\pi^{-}, K^{-}$, and $\bar{p}$, (b) $\pi^{+}, K^{+}$, and $p$, (c) $\pi^{+}$, (d) $K^{+}$, and (e) $p$ produced in $p p$ collision at $\sqrt{s}=200 \mathrm{GeV}$ with different $y$ ranges and magnifications are shown in the figure. The symbols represent the experimental data of the STAR [26] and BRAHMS Collaborations [27] and the curves are our results calculated by the Boltzmann or two-component Boltzmann distribution.

the considered distribution is described by a multicomponent Erlang distribution $[19,20]$. Different from some simulation codes, our model gives directly a few statistical laws by analytical expressions in describing some quantities. In the case of being incapable of analytical expressions, we could use a Monte Carlo method to give a numerical result. Our model is easy to be used by experimental experts.

Due to significances of the considered model and topic, in this paper, based on Boltzmann distribution for a single source, we describe $p_{T}$ spectra of final-state particles 


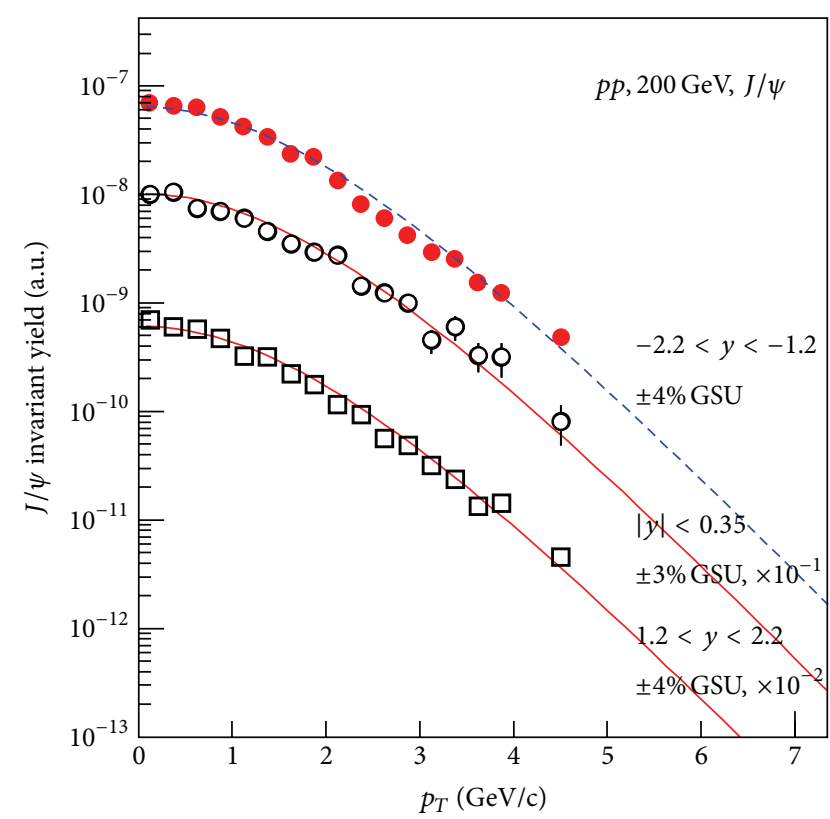

(a)

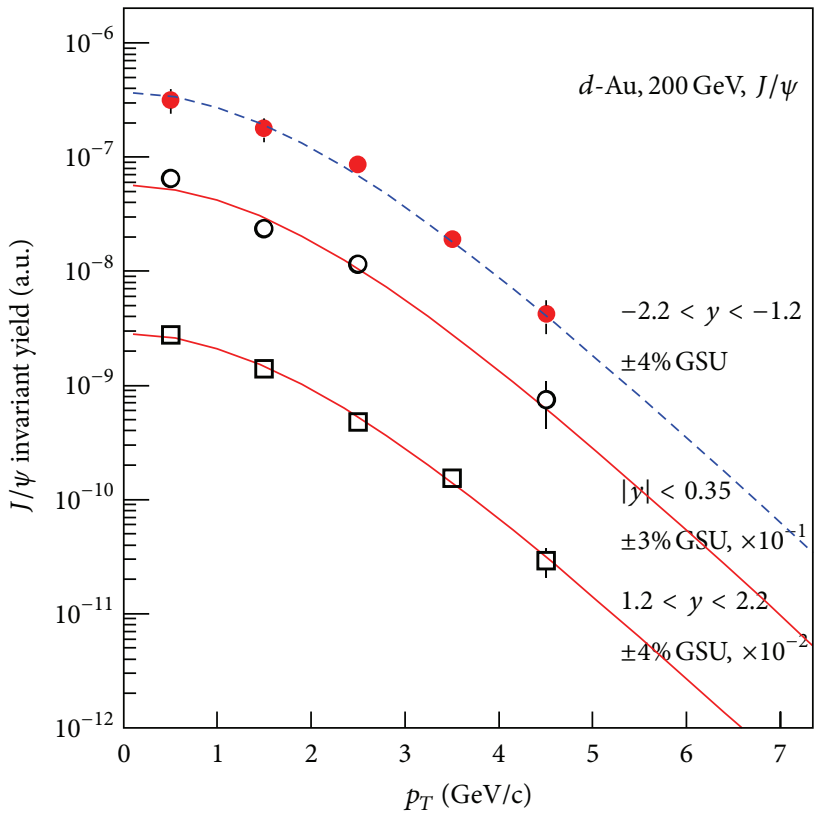

(c)

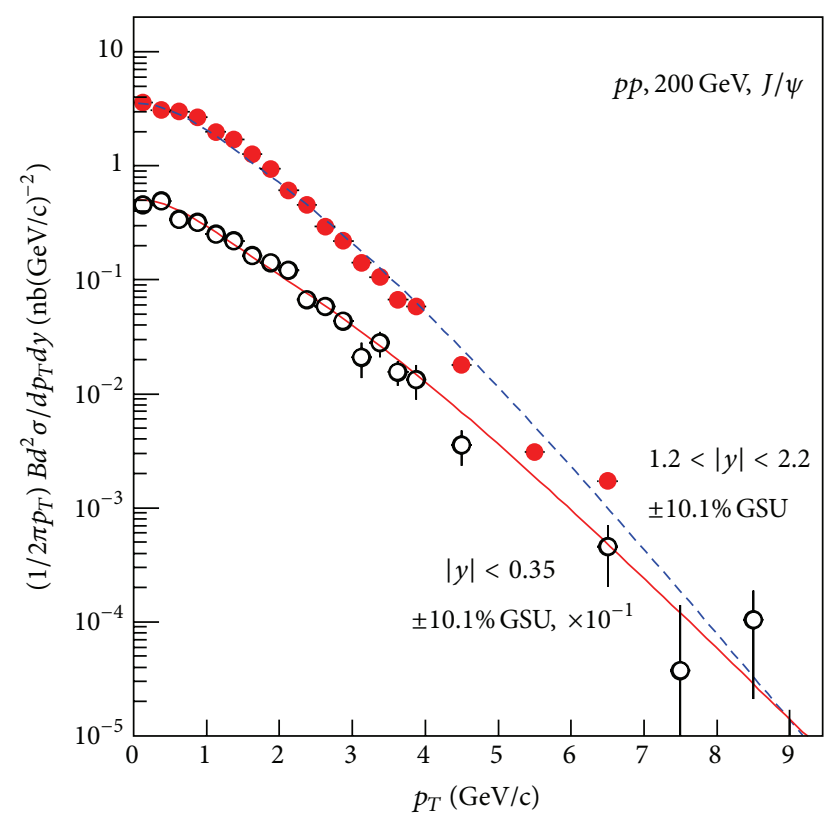

(b)

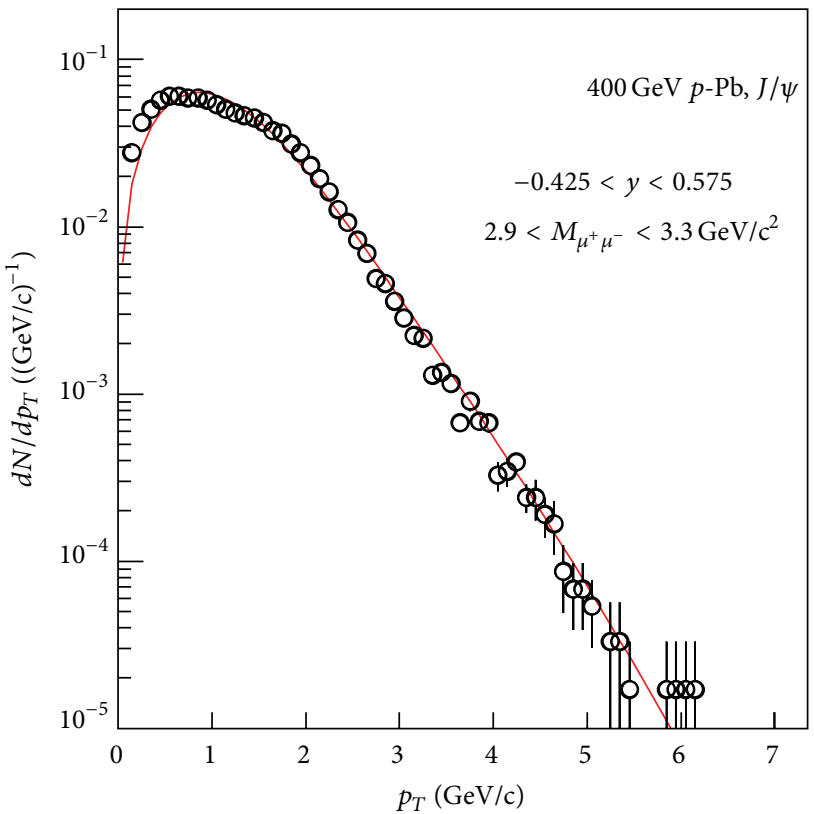

(d)

Figure 2: The transverse momentum distributions of $J / \psi$ produced in (a) and (b) $p p$ collision at $\sqrt{s}=200 \mathrm{GeV}$, (c) $d$-Au collisions at $\sqrt{s_{N N}}=200 \mathrm{GeV}$, and (d) $p-\mathrm{Pb}$ collisions at beam energy being $400 \mathrm{GeV}$ with different $y$ ranges, $\mathrm{GSU}$ (or $M_{\mu^{+} \mu^{-}}$) ranges, and magnifications are shown in the figure. The symbols represent the experimental data of the PHENIX [28-30] and NA50 Collaborations [31] and the curves are our results calculated by the Boltzmann or two-component Boltzmann distribution.

produced in soft process in proton-proton $(p P)$ and nucleusnucleus $(A A)$ collisions at RHIC and LHC energies. Some interesting results are obtained.

\section{The Model}

According to the multisource thermal model, many emission sub-sources of final-state particles are assumed to form in high energy collisions $[19,20]$. These multiple sub-sources can be different regions in the overlap region or different mechanisms, and these particles can be created/emitted at different times in the collisions. In fact, these sub-sources can be divided into different groups (sources) due to different interacting mechanisms or event samples. Obviously, soft process corresponds to sources with low degree of excitation or to particles with low transverse momentum, and hard process corresponds to sources with high degree of excitation or to particles with high transverse momentum, 


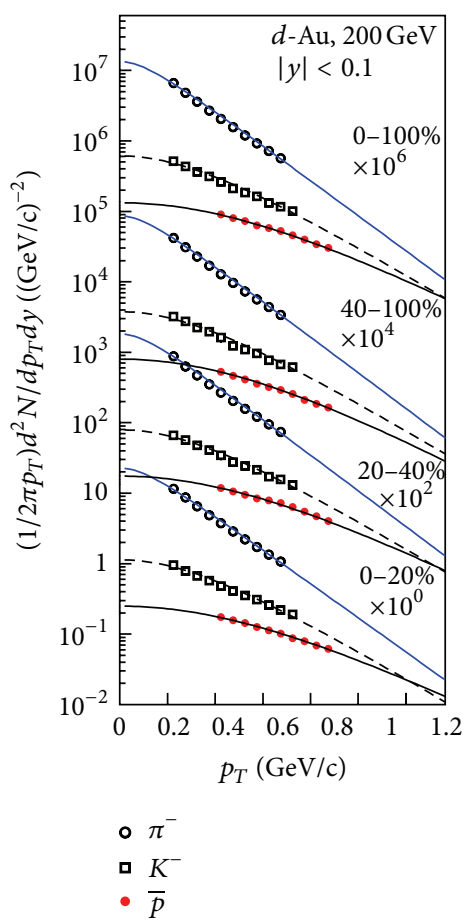

(a)

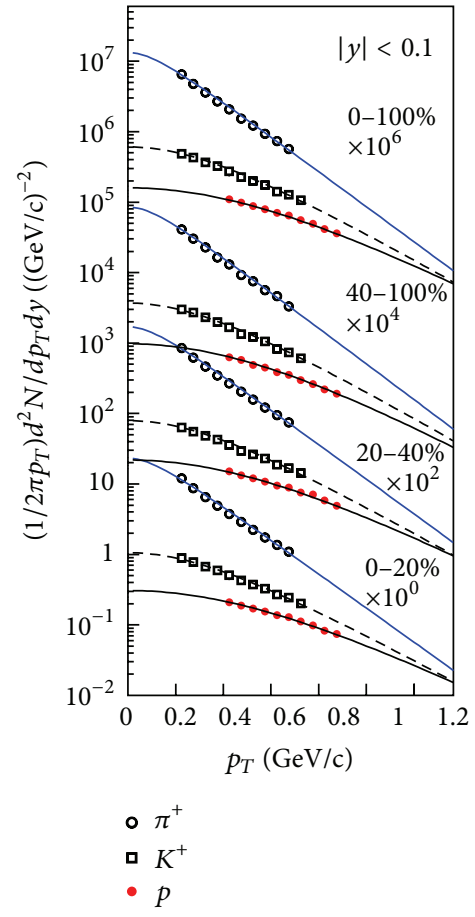

(b)

FIGURE 3: The transverse momentum distributions of (a) $\pi^{-}, K^{-}$, and $\bar{p}$ as well as (b) $\pi^{+}, K^{+}$, and $p$ produced in $d$-Au collisions at $\sqrt{s_{N N}}=$ $200 \mathrm{GeV}$ with $|y|<0.1$ and different centrality classes and magnifications are shown in the figure. The symbols represent the experimental data of the STAR Collaboration [26] and the curves are our results calculated by the Boltzmann or two-component Boltzmann distribution.

TABLE 1: Parameter values corresponding to the curves in Figure 1.

\begin{tabular}{|c|c|c|c|c|c|c|c|}
\hline Figure & Collision & Particle & Rapidity & $T_{1}(\mathrm{GeV})$ & $k_{1}$ & $T_{2}(\mathrm{GeV})$ & $\chi^{2} /$ dof \\
\hline \multirow{3}{*}{ Figure 1(a)/Figure 1(b) } & \multirow{3}{*}{$p p 200 \mathrm{GeV}$} & $\pi^{-} / \pi^{+}$ & $|y|<0.1$ & 0.165 & 1.000 & - & $1.128 / 1.212$ \\
\hline & & $K^{-} / K^{+}$ & $|y|<0.1$ & 0.187 & 1.000 & - & $0.211 / 0.243$ \\
\hline & & $\bar{p} / p$ & $|y|<0.1$ & $0.197 / 0.212$ & 1.000 & - & $0.340 / 0.274$ \\
\hline \multirow{7}{*}{ Figure $1(\mathrm{c})$} & \multirow{7}{*}{$p p 200 \mathrm{GeV}$} & \multirow{7}{*}{$\pi^{+}$} & $0.00 \pm 0.10$ & 0.175 & 0.896 & 0.360 & 0.849 \\
\hline & & & $0.90 \pm 0.10$ & 0.175 & 0.930 & 0.360 & 0.958 \\
\hline & & & $1.20 \pm 0.10$ & 0.175 & 0.912 & 0.360 & 0.968 \\
\hline & & & $2.95 \pm 0.05$ & 0.175 & 0.823 & 0.270 & 1.415 \\
\hline & & & $3.00 \pm 0.10$ & 0.175 & 0.856 & 0.270 & 1.170 \\
\hline & & & $3.30 \pm 0.10$ & 0.166 & 0.888 & 0.270 & 1.669 \\
\hline & & & $3.50 \pm 0.10$ & 0.205 & 1.000 & - & 1.273 \\
\hline \multirow{7}{*}{ Figure 1(d) } & \multirow{7}{*}{ pp $200 \mathrm{GeV}$} & \multirow{7}{*}{$K^{+}$} & $0.00 \pm 0.10$ & 0.160 & 0.700 & 0.360 & 0.499 \\
\hline & & & $0.90 \pm 0.10$ & 0.160 & 0.600 & 0.360 & 0.786 \\
\hline & & & $1.20 \pm 0.10$ & 0.160 & 0.600 & 0.360 & 0.437 \\
\hline & & & $2.95 \pm 0.05$ & 0.198 & 0.800 & 0.360 & 1.048 \\
\hline & & & $3.00 \pm 0.10$ & 0.176 & 0.788 & 0.300 & 0.758 \\
\hline & & & $3.30 \pm 0.10$ & 0.160 & 0.780 & 0.273 & 1.689 \\
\hline & & & $3.50 \pm 0.10$ & 0.160 & 0.667 & 0.253 & 0.714 \\
\hline \multirow{7}{*}{ Figure 1(e) } & \multirow{7}{*}{$p p 200 \mathrm{GeV}$} & \multirow{7}{*}{$p$} & $0.00 \pm 0.10$ & 0.160 & 0.400 & 0.265 & 1.028 \\
\hline & & & $0.90 \pm 0.10$ & 0.160 & 0.540 & 0.290 & 1.240 \\
\hline & & & $1.20 \pm 0.10$ & 0.160 & 0.540 & 0.290 & 0.821 \\
\hline & & & $2.95 \pm 0.05$ & 0.156 & 0.875 & 0.280 & 1.457 \\
\hline & & & $3.00 \pm 0.10$ & 0.156 & 0.872 & 0.280 & 0.750 \\
\hline & & & $3.30 \pm 0.10$ & 0.190 & 0.859 & 0.280 & 1.561 \\
\hline & & & $3.50 \pm 0.10$ & 0.176 & 1.000 & - & 0.907 \\
\hline
\end{tabular}




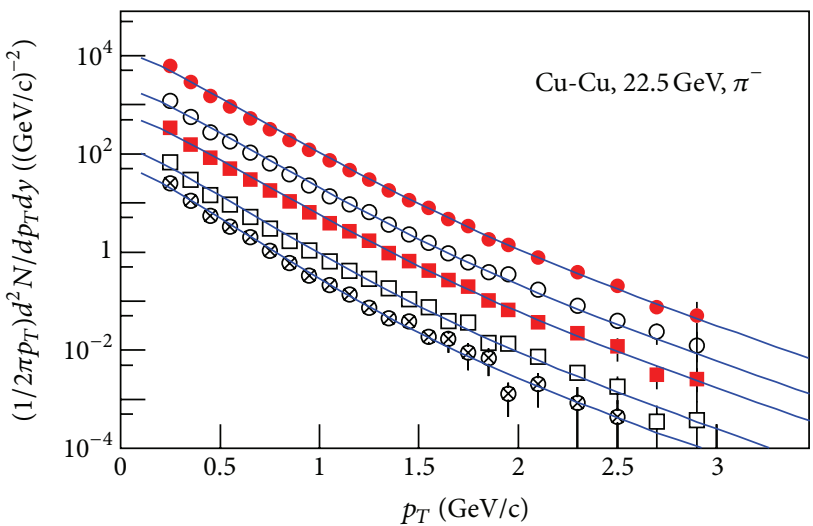

(a)

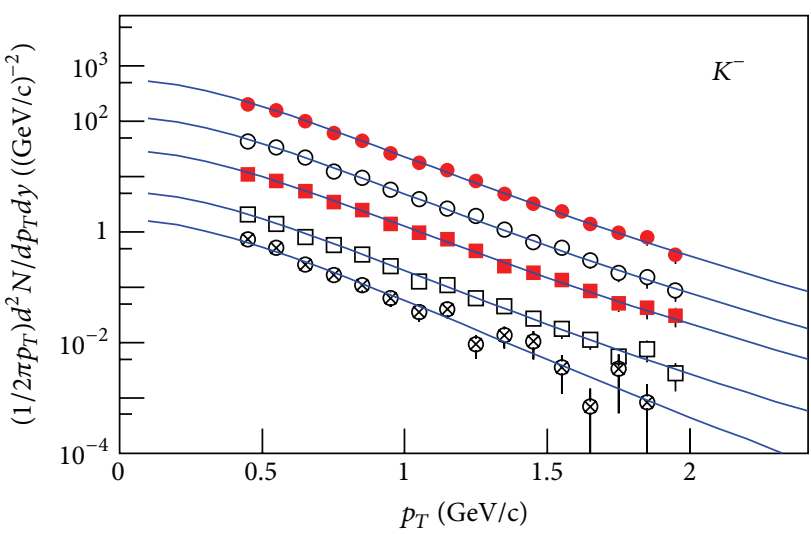

(c)

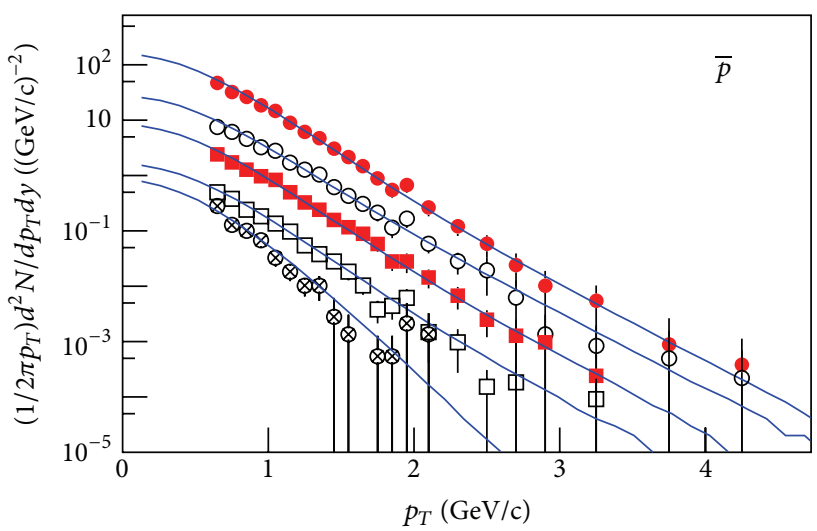

- $0-100 \%, \times 300$

○ $0-10 \%, \times 20$

- $10-30 \%, \times 10$

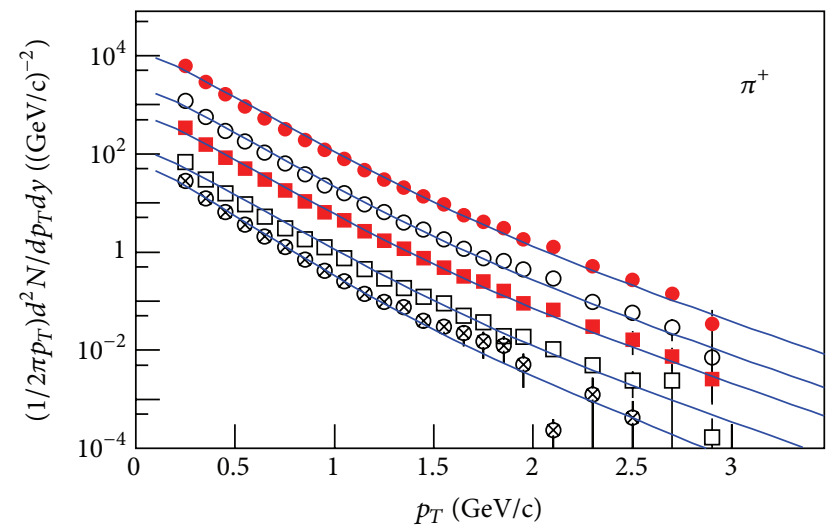

(b)

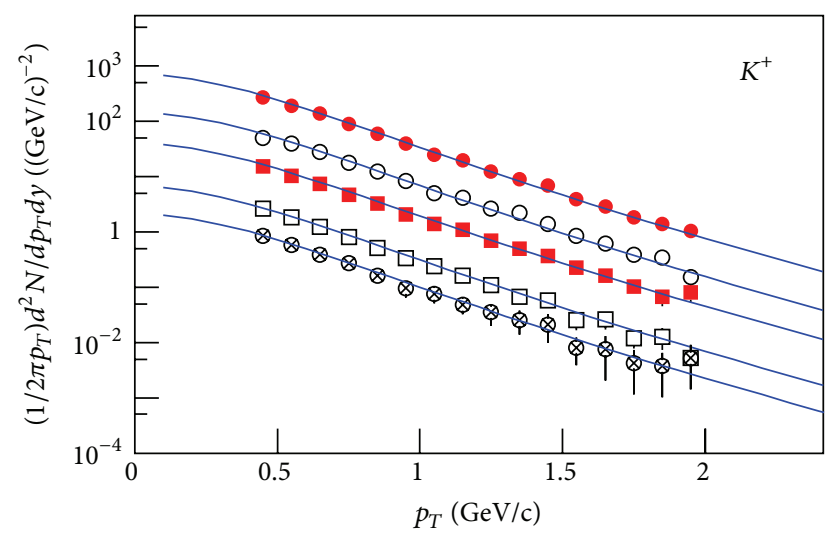

(d)

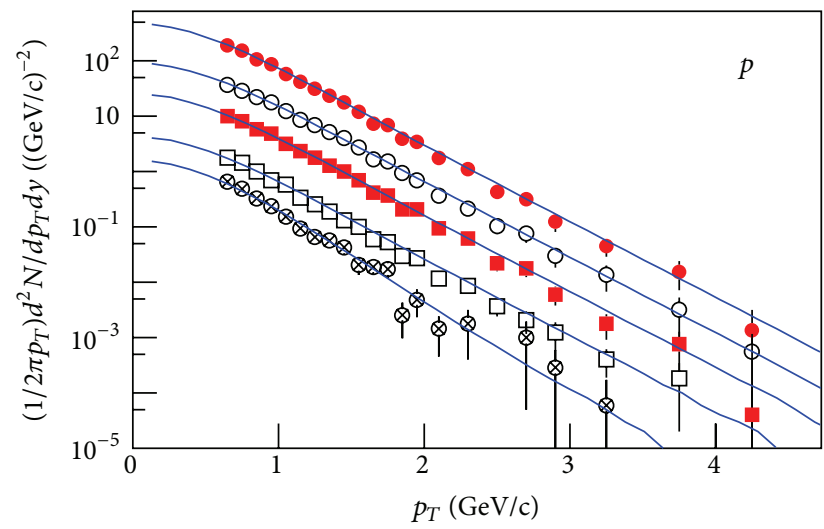

$\begin{array}{ll} & 0-100 \%, \times 300 \\ & 0-10 \%, \times 20 \\ & 10-30 \%, \times 10\end{array}$

ㅁ 30-60\%, $\times 5$

$\otimes 60-100 \%, \times 10$

(f)

Figure 4: The transverse momentum distributions of (a) $\pi^{-}$, (b) $\pi^{+}$, (c) $K^{-}$, (d) $K^{+}$, (e) $\bar{p}$, and (f) $p$ produced in Cu-Cu collisions at $\sqrt{s_{N N}}=$ $22.5 \mathrm{GeV}$ with different centrality classes and magnifications are shown in the figure. The symbols represent the experimental data of the PHENIX Collaboration [32] and the curves are our results calculated by the Boltzmann or two-component Boltzmann distribution.

where the excitation means to create particles through string breaking, direct scattering, recombination, and their hybrid.

In the rest frame of a source, we consider the source as a thermodynamic system of relativistic and quantum ideal gas. The momentum $(p)$ distribution of final-state particles in the natural unit system is given by [21]

$$
f_{p}(p)=\frac{1}{N} \frac{d N}{d p}=C_{0} p^{2}\left[\exp \left(\frac{\sqrt{p^{2}+m_{0}^{2}}-\mu}{T}\right) \pm 1\right]^{-1}
$$

where $N$ is the number of particles, $C_{0}$ is the normalization constant, $m_{0}$ is the rest mass of a considered particle, $\mu$ 
TABLE 2: Parameter values corresponding to the curves in Figure 2.

\begin{tabular}{|c|c|c|c|c|c|c|c|}
\hline Figure & Collision & Particle & Rapidity & $T_{1}(\mathrm{GeV})$ & $k_{1}$ & $T_{2}(\mathrm{GeV})$ & $\chi^{2} /$ dof \\
\hline \multirow{3}{*}{ Figure 2(a) } & \multirow{3}{*}{$p p 200 \mathrm{GeV}$} & \multirow{3}{*}{$J / \psi$ in $p p$} & $-2.2<y<-1.2$ & 0.462 & 1.000 & - & 0.663 \\
\hline & & & $|y|<0.35$ & 0.462 & 1.000 & - & 1.157 \\
\hline & & & $1.2<y<2.2$ & 0.462 & 1.000 & - & 1.383 \\
\hline \multirow{2}{*}{ Figure 2(b) } & \multirow{2}{*}{$p p 200 \mathrm{GeV}$} & \multirow{2}{*}{$J / \psi$ in $p p$} & $1.2<|y|<2.2$ & 0.160 & 0.175 & 0.541 & 1.371 \\
\hline & & & $|y|<0.35$ & 0.160 & 0.175 & 0.654 & 1.638 \\
\hline \multirow{3}{*}{ Figure 2(c) } & \multirow{3}{*}{$d$-Au $200 \mathrm{GeV}$} & \multirow{3}{*}{$J / \psi$ in $d-\mathrm{Au}$} & $-2.2<y<-1.2$ & 0.525 & 1.000 & - & 1.332 \\
\hline & & & $|y|<0.35$ & 0.525 & 1.000 & - & 1.125 \\
\hline & & & $1.2<y<2.2$ & 0.525 & 1.000 & 一 & 1.845 \\
\hline Figure 2(d) & $400 \mathrm{GeV} p-\mathrm{Pb}$ & $J / \psi$ in $p-\mathrm{Pb}$ & $-0.425<y<0.575$ & 0.205 & 0.650 & 0.368 & 1.041 \\
\hline
\end{tabular}

TABlE 3: Parameter values corresponding to the curves in Figure 3(a)/Figure 3(b). The collisions are $d+\mathrm{Au}$ at $200 \mathrm{GeV}$.

\begin{tabular}{lccc}
\hline Particle & Centrality & $T_{1}(\mathrm{GeV})$ & $\chi^{2} / \mathrm{dof}$ \\
\hline & $0-20 \%$ & 0.178 & $0.564 / 0.704$ \\
$\pi^{-} / \pi^{+}$ & $20-40 \%$ & $0.170 / 0.175$ & $1.053 / 0.779$ \\
& $40-100 \%$ & 0.170 & $0.879 / 0.905$ \\
& $0-100 \%$ & 0.173 & $0.796 / 0.771$ \\
\hline & $0-20 \%$ & $0.206 / 0.228$ & $0.119 / 0.052$ \\
$K^{-} / K^{+}$ & $20-40 \%$ & $0.206 / 0.218$ & $0.112 / 0.114$ \\
& $40-100 \%$ & $0.206 / 0.213$ & $0.216 / 0.074$ \\
& $0-100 \%$ & $0.206 / 0.217$ & $0.137 / 0.131$ \\
\hline & $0-20 \%$ & $0.243 / 0.239$ & $0.036 / 0.032$ \\
$\bar{p} / p$ & $20-40 \%$ & $0.232 / 0.229$ & $0.047 / 0.036$ \\
& $40-100 \%$ & $0.215 / 0.212$ & $0.091 / 0.082$ \\
& $0-100 \%$ & 0.229 & $0.032 / 0.013$ \\
\hline
\end{tabular}

is the chemical potential, $T$ is the temperature parameter, +1 denotes fermions, and -1 denotes bosons, respectively. Our calculations show that at RHIC and LHC energies the quantum effect and chemical potential can be neglected compared to the relativistic effect [22]. Then, we have a simple expression for momentum distribution to be $[23,24]$

$$
f_{p}(p)=\frac{1}{N} \frac{d N}{d p}=C_{0} p^{2} \exp \left(-\frac{\sqrt{p^{2}+m_{0}^{2}}}{T}\right)
$$

where $C_{0}=\left(1 / m_{0}^{2} T\right)\left(1 / K_{2}\left(m_{0} / T\right)\right)$ and $K_{2}\left(m_{0} / T\right)$ is the modified Bessel function of order 2 .

The $p_{T}$ distribution can be written as a Boltzmann distribution [25]:

$$
f_{p_{T}}\left(p_{T}\right)=\frac{1}{N} \frac{d N}{d p_{T}}=C p_{T} \exp \left(-\frac{\sqrt{p_{T}^{2}+m_{0}^{2}}}{T}\right),
$$

where $C$ is the normalization constant. Because of interactions among different sources, the considered source has a deformation and/or movement in the transverse plane. Let $a$ denote a relative deformation and let $b$ denote an absolute movement of the source; that is, we use $a p_{T}+b$ instead of $p_{T}$ in (3). The revised $p_{T}$ distribution can be given by

$$
\begin{aligned}
f_{p_{T}}\left(p_{T}\right) & =\frac{1}{N} \frac{d N}{d p_{T}} \\
& =C\left(p_{T}-b\right) a^{-2} \exp \left[-\frac{\sqrt{\left(p_{T}-b\right)^{2} a^{-2}+m_{0}^{2}}}{T}\right] .
\end{aligned}
$$

In the case of considering multiple sources, we have

$$
f_{p_{T}}\left(p_{T}\right)=\frac{1}{N} \frac{d N}{d p_{T}}=\sum_{i=1}^{n} k_{i} C_{i} p_{T} \exp \left(-\frac{\sqrt{p_{T}^{2}+m_{0}^{2}}}{T_{i}}\right)
$$

or

$$
\begin{aligned}
& f_{p_{T}}\left(p_{T}\right) \\
& =\frac{1}{N} \frac{d N}{d p_{T}} \\
& =\sum_{i=1}^{n} k_{i} C_{i}\left(p_{T}-b_{i}\right) a_{i}^{-2} \exp \left[-\frac{\sqrt{\left(p_{T}-b_{i}\right)^{2} a_{i}^{-2}+m_{0}^{2}}}{T_{i}}\right],
\end{aligned}
$$

where $k_{i}, C_{i}$, and $T_{i}$ denote the contribution ratio, normalization constant, and temperature of the ith source, respectively. Because the effects of deformation and movement of the source can be neglected in the calculation of transverse momentum, we take the default values of $a_{i}=1$ and $b_{i}=0$ in the revised $p_{T}$ distribution, which results from the Boltzmann distribution or a multicomponent Boltzmann distribution.

We should have a few sources to describe the soft and hard processes. For the soft process, the number of sources is generally 1 or 2 . For the hard process, the number of sources is also 1 or 2 . The total number of sources will be from 2 to 4 for a wide $p_{T}$ distribution. In this paper, we pay our attention on the soft process which has a narrow $p_{T}$ distribution. It is hard to say that what the $p_{T}$ distribution range is for the soft process. What we can say is that for low energy collisions the distribution range is narrower. In the present work, we regard the distribution range as $0-10 \mathrm{GeV} / \mathrm{c}$. The difference 
TABLE 4: Parameter values corresponding to the curves in Figure 4.

\begin{tabular}{|c|c|c|c|c|c|c|c|}
\hline Figure & Collision & Particle & Centrality & $T_{1}(\mathrm{GeV})$ & $k_{1}$ & $T_{2}(\mathrm{GeV})$ & $\chi^{2} /$ dof \\
\hline \multirow{5}{*}{ Figure 4(a)/Figure 4(b) } & \multirow{5}{*}{$\begin{array}{c}\mathrm{Cu}-\mathrm{Cu} \\
22.5 \mathrm{GeV}\end{array}$} & \multirow{5}{*}{$\pi^{-} / \pi^{+}$} & $0-10 \%$ & 0.183 & $0.934 / 0.912$ & 0.310 & $1.079 / 1.008$ \\
\hline & & & $10-30 \%$ & 0.183 & $0.934 / 0.917$ & 0.310 & $1.195 / 0.848$ \\
\hline & & & $30-60 \%$ & $0.173 / 0.183$ & $0.934 / 0.931$ & $0.300 / 0.310$ & $0.992 / 1.810$ \\
\hline & & & $60-100 \%$ & $0.162 / 0.160$ & $0.934 / 0.900$ & $0.293 / 0.270$ & $1.394 / 0.959$ \\
\hline & & & $0-100 \%$ & 0.183 & $0.934 / 0.920$ & 0.310 & $1.229 / 1.084$ \\
\hline \multirow{5}{*}{ Figure 4(c)/Figure 4(d) } & \multirow{5}{*}{$\begin{array}{c}\mathrm{Cu}-\mathrm{Cu} \\
22.5 \mathrm{GeV}\end{array}$} & \multirow{5}{*}{$K^{-} / K^{+}$} & $0-10 \%$ & 0.183 & $0.860 / 0.747$ & 0.310 & $0.636 / 0.593$ \\
\hline & & & $10-30 \%$ & 0.183 & $0.840 / 0.732$ & 0.310 & $0.632 / 0.818$ \\
\hline & & & $30-60 \%$ & 0.183 & $0.900 / 0.755$ & 0.310 & $1.087 / 1.440$ \\
\hline & & & $60-100 \%$ & 0.183 & $0.975 / 0.747$ & 0.310 & $1.677 / 1.311$ \\
\hline & & & $0-100 \%$ & 0.183 & $0.860 / 0.747$ & 0.310 & $0.632 / 0.515$ \\
\hline \multirow{5}{*}{ Figure 4(e)/Figure 4(f) } & \multirow{5}{*}{$\begin{array}{l}\mathrm{Cu}-\mathrm{Cu} \\
22.5 \mathrm{GeV}\end{array}$} & \multirow{5}{*}{$\bar{p} / p$} & $0-10 \%$ & $0.183 / 0.200$ & $0.760 / 0.510$ & 0.310 & $1.898 / 0.904$ \\
\hline & & & $10-30 \%$ & $0.183 / 0.200$ & $0.860 / 0.567$ & 0.310 & $0.825 / 1.159$ \\
\hline & & & $30-60 \%$ & $0.183 / 0.200$ & $0.883 / 0.580$ & 0.310 & $1.849 / 1.747$ \\
\hline & & & $60-100 \%$ & $0.160 / 0.200$ & $1.000 / 0.888$ & $-/ 0.310$ & $1.953 / 1.855$ \\
\hline & & & $0-100 \%$ & $0.183 / 0.200$ & $0.862 / 0.566$ & 0.310 & $1.090 / 1.466$ \\
\hline
\end{tabular}

TABle 5: Parameter values corresponding to the curves in Figure 5(a)/Figure 5(b). The collisions are $\mathrm{Au}+\mathrm{Au}$ at $62.4 \mathrm{GeV}$.

\begin{tabular}{|c|c|c|c|}
\hline Particle & Centrality & $T_{1}(\mathrm{GeV})$ & $\chi^{2} /$ dof \\
\hline \multirow{9}{*}{$\pi^{-} / \pi^{+}$} & $0-5 \%$ & 0.185 & $0.184 / 0.184$ \\
\hline & $5-10 \%$ & 0.185 & $0.192 / 0.156$ \\
\hline & $10-20 \%$ & 0.182 & $0.224 / 0.246$ \\
\hline & $20-30 \%$ & 0.180 & $0.230 / 0.228$ \\
\hline & $30-40 \%$ & 0.180 & $0.276 / 0.302$ \\
\hline & $40-50 \%$ & 0.175 & $0.112 / 0.128$ \\
\hline & $50-60 \%$ & 0.170 & $0.118 / 0.146$ \\
\hline & $60-70 \%$ & 0.170 & $0.138 / 0.234$ \\
\hline & $70-80 \%$ & 0.165 & $0.254 / 0.292$ \\
\hline \multirow{9}{*}{$K^{-} / K^{+}$} & $0-5 \%$ & 0.275 & $0.056 / 0.010$ \\
\hline & $5-10 \%$ & 0.275 & $0.024 / 0.018$ \\
\hline & $10-20 \%$ & 0.275 & $0.040 / 0.018$ \\
\hline & $20-30 \%$ & 0.253 & $0.064 / 0.046$ \\
\hline & $30-40 \%$ & 0.243 & $0.102 / 0.154$ \\
\hline & $40-50 \%$ & 0.235 & $0.082 / 0.132$ \\
\hline & $50-60 \%$ & 0.215 & $0.166 / 0.084$ \\
\hline & $60-70 \%$ & 0.205 & $0.100 / 0.080$ \\
\hline & $70-80 \%$ & 0.205 & $0.362 / 0.130$ \\
\hline \multirow{9}{*}{$\bar{p} / p$} & $0-5 \%$ & 0.512 & $0.114 / 0.166$ \\
\hline & $5-10 \%$ & 0.475 & $0.068 / 0.120$ \\
\hline & $10-20 \%$ & 0.442 & $0.048 / 0.096$ \\
\hline & $20-30 \%$ & 0.393 & $0.040 / 0.136$ \\
\hline & $30-40 \%$ & 0.355 & $0.076 / 0.082$ \\
\hline & $40-50 \%$ & 0.305 & $0.104 / 0.080$ \\
\hline & $50-60 \%$ & 0.270 & $0.070 / 0.086$ \\
\hline & $60-70 \%$ & 0.250 & $0.038 / 0.058$ \\
\hline & $70-80 \%$ & 0.220 & $0.020 / 0.084$ \\
\hline
\end{tabular}

between the single and multisource models is obvious. The former one describes a narrower distribution which
TABle 6: Parameter values corresponding to the curves in Figure 6(a)/Figure 6(b). The collisions are $\mathrm{Au}+\mathrm{Au}$ at $130 \mathrm{GeV}$.

\begin{tabular}{cccc}
\hline Particle & Centrality & $T_{1}(\mathrm{GeV})$ & $\chi^{2} /$ dof \\
\hline $0-6 \%$ & 0.185 & $0.210 / 0.268$ \\
$6-11 \%$ & 0.182 & $0.290 / 0.232$ \\
$\pi^{-} / \pi^{+}$ & $11-18 \%$ & 0.180 & $0.430 / 0.328$ \\
& $18-26 \%$ & 0.180 & $0.394 / 0.310$ \\
& $26-34 \%$ & 0.175 & $0.222 / 0.172$ \\
& $34-45 \%$ & 0.170 & $0.126 / 0.144$ \\
& $45-58 \%$ & 0.170 & $0.192 / 0.234$ \\
$K^{-} / K^{+}$ & $58-85 \%$ & 0.165 & $0.334 / 0.354$ \\
\hline & $0-6 \%$ & 0.275 & $0.088 / 0.146$ \\
& $6-11 \%$ & 0.275 & $0.268 / 0.087$ \\
$11-18 \%$ & $0.273 / 0.263$ & $0.151 / 0.584$ \\
& $18-26 \%$ & 0.283 & $0.189 / 0.584$ \\
& $26-34 \%$ & $0.255 / 0.235$ & $0.101 / 0.598$ \\
& $34-45 \%$ & $0.260 / 0.245$ & $0.846 / 0.293$ \\
& $45-58 \%$ & $0.245 / 0.205$ & $0.639 / 0.448$ \\
& $58-85 \%$ & 0.205 & $0.311 / 0.543$ \\
\hline $0-6 \%$ & 0.580 & $0.177 / 0.177$ \\
& $6-11 \%$ & 0.530 & $0.157 / 0.128$ \\
& $11-18 \%$ & 0.450 & $0.123 / 0.104$ \\
$18-26 \%$ & 0.410 & $0.105 / 0.118$ \\
$26-34 \%$ & 0.410 & $0.103 / 0.068$ \\
& $34-45 \%$ & 0.363 & $0.071 / 0.121$ \\
& $45-58 \%$ & 0.303 & $0.076 / 0.065$ \\
$58-85 \%$ & 0.265 & $0.029 / 0.055$ \\
\hline
\end{tabular}

corresponds to an equilibrium state with a lower degree of excitation. The latter one describes a wider distribution which corresponds to a few local equilibrium sates with different excitations. 


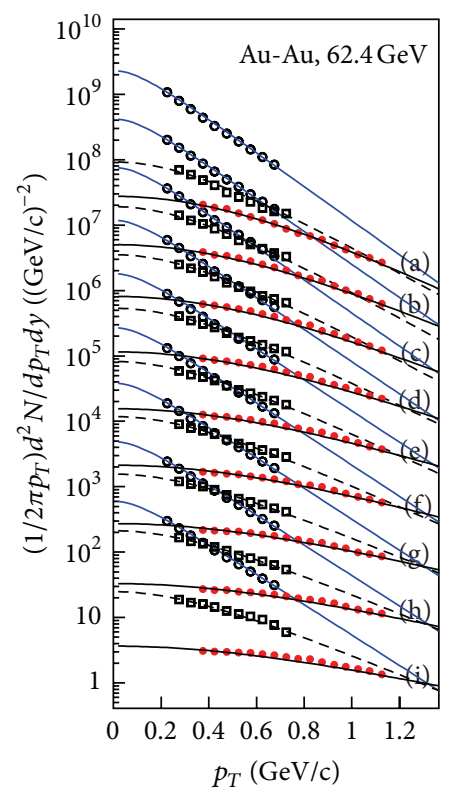
(a) $70-80 \%, \times 10^{8}$
(b) $60-70 \%, \times 10^{7}$
(c) $50-60 \%, \times 10^{6}$
(d) $40-50 \%, \times 10^{5}$
○ $\pi^{-}$
व $K^{-}$
- $\bar{p}$

(a)

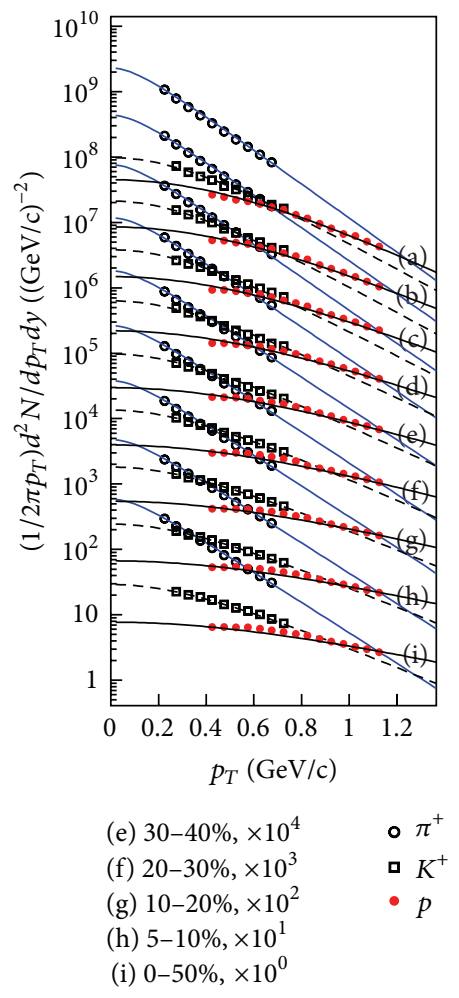

(b)

FIgURE 5: The transverse momentum distributions of (a) $\pi^{-}, K^{-}$, and $\bar{p}$, and (b) $\pi^{+}, K^{+}$, and $p$ produced in Au-Au collisions at $\sqrt{s_{N N}}=$ $62.4 \mathrm{GeV}$ with different centrality classes and magnifications are shown in the figure. The symbols represent the experimental data of the STAR Collaboration [26] and the curves are our results calculated by the Boltzmann or two-component Boltzmann distribution.
TABLE 7: Parameter values corresponding to the curves in Figure 7(a)/Figure 7(b). The collisions are $\mathrm{Au}+\mathrm{Au}$ at $200 \mathrm{GeV}$.

\begin{tabular}{|c|c|c|c|}
\hline Particle & Centrality & $T_{1}(\mathrm{GeV})$ & $\chi^{2} /$ dof \\
\hline \multirow{9}{*}{$\pi^{-} / \pi^{+}$} & $0-5 \%$ & 0.193 & $0.940 / 0.746$ \\
\hline & $5-10 \%$ & 0.193 & $0.865 / 0.907$ \\
\hline & $10-20 \%$ & $0.193 / 0.190$ & $0.780 / 0.444$ \\
\hline & $20-30 \%$ & $0.190 / 0.188$ & $0.628 / 0.661$ \\
\hline & $30-40 \%$ & 0.188 & $1.432 / 1.449$ \\
\hline & $40-50 \%$ & 0.188 & $0.570 / 0.577$ \\
\hline & $50-60 \%$ & 0.180 & $0.974 / 0.914$ \\
\hline & $60-70 \%$ & 0.175 & $1.269 / 1.295$ \\
\hline & $70-80 \%$ & 0.170 & $1.269 / 1.311$ \\
\hline \multirow{9}{*}{$K^{-} / K^{+}$} & $0-5 \%$ & 0.327 & $0.019 / 0.020$ \\
\hline & $5-10 \%$ & 0.327 & $0.019 / 0.032$ \\
\hline & $10-20 \%$ & 0.285 & $0.044 / 0.034$ \\
\hline & $20-30 \%$ & 0.285 & $0.029 / 0.022$ \\
\hline & $30-40 \%$ & 0.243 & $0.112 / 0.107$ \\
\hline & $40-50 \%$ & 0.243 & $0.082 / 0.079$ \\
\hline & $50-60 \%$ & 0.243 & $0.007 / 0.021$ \\
\hline & $60-70 \%$ & 0.215 & $0.081 / 0.071$ \\
\hline & $70-80 \%$ & 0.200 & $0.235 / 0.143$ \\
\hline \multirow{9}{*}{$\bar{p} / p$} & $0-5 \%$ & 0.570 & $0.046 / 0.031$ \\
\hline & $5-10 \%$ & 0.516 & $0.071 / 0.013$ \\
\hline & $10-20 \%$ & 0.516 & $0.037 / 0.045$ \\
\hline & $20-30 \%$ & 0.435 & $0.047 / 0.035$ \\
\hline & $30-40 \%$ & 0.396 & $0.039 / 0.036$ \\
\hline & $40-50 \%$ & $0.338 / 0.345$ & $0.035 / 0.032$ \\
\hline & $50-60 \%$ & $0.312 / 0.320$ & $0.025 / 0.031$ \\
\hline & $60-70 \%$ & 0.270 & $0.086 / 0.057$ \\
\hline & $70-80 \%$ & 0.246 & $0.045 / 0.022$ \\
\hline
\end{tabular}

\section{Comparisons with Experimental Data}

Figure 1 presents the transverse momentum distributions of (a) $\pi^{-}, K^{-}$and $\bar{p}$, (b) $\pi^{+}, K^{+}$and $p$, (c) $\pi^{+}$, (d) $K^{+}$, and (e) $p$ produced in $p p$ collision at center-of-mass energy $\sqrt{s}=$ $200 \mathrm{GeV}$ with different $y$ ranges and magnifications shown in the figure. The symbols represent the experimental data of the STAR [26] (Figures 1(a) and 1(b)) and BRAHMS Collaborations [27] (Figures 1(c)-1(e)), and the curves are our results calculated by the Boltzmann or two-component Boltzmann distribution. In the calculation, we have used a fitting method to obtain parameter values which are shown in Table 1 with values of $\chi^{2}$ per degree of freedom ( $\chi^{2} /$ dof). To give a short presentation, the values corresponding to "negative/positive" charged particles are given in terms of "the first value/the second value" or "value" in the case of the first value and the second value being the same. We would like to point out that the presenting styles of rapidity ranges for Figures 1(a) and 1(b) as well as for Figures 1(c)-1(e) are different due to different presentations in $[26,27]$. One can see that the modeling results with 1 or 2 sources are in agreement with the experimental data. For emissions of $\pi^{-} / \pi^{+}, K^{-} / K^{+}$and $\bar{p} / p$, the temperature parameter increases with increase of particle mass (Figures $1(\mathrm{a})$ and $1(\mathrm{~b})$ ), which indicates the impact of 


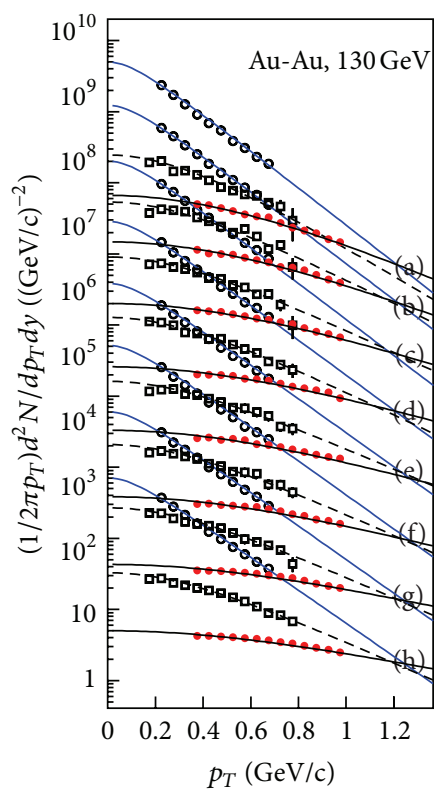
(a) $58-85 \%, \times 10^{7}$
(b) $45-58 \%, \times 10^{6}$
(c) $34-45 \%, \times 10^{5}$

(d) $26-34 \%, \times 10^{4}$

(a)

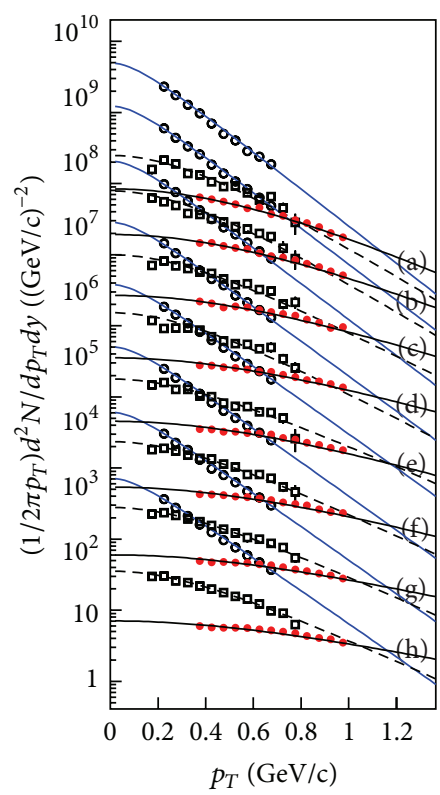

$\begin{array}{ll}\text { (e) } 18-26 \%, \times 10^{3} & \text { } \pi^{+} \\ \text {(f) } 11-18 \%, \times 10^{2} & \text { } K^{+} \\ \text {(g) } 6-11 \%, \times 10^{1} & \text { - } p\end{array}$

(h) $0-6 \%, \times 10^{0}$

(b)

FIGURE 6: The same as Figure 5, but showing the results at $\sqrt{s_{N N}}=130 \mathrm{GeV}$.

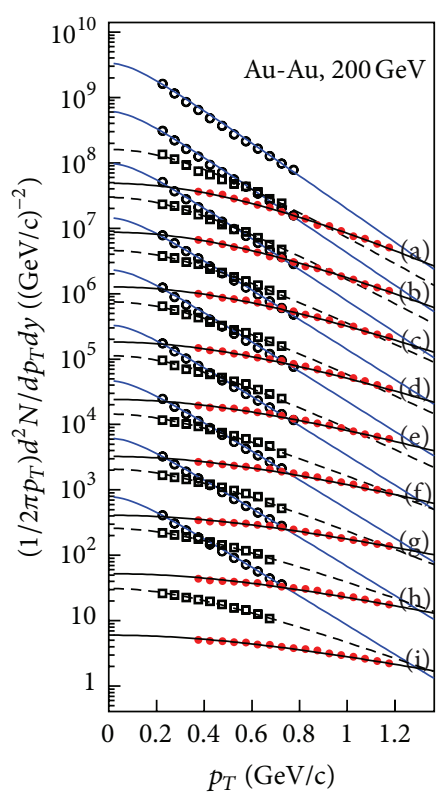

(a) $70-80 \%, \times 10^{8}$ (b) $60-70 \%, \times 10^{7}$

(c) $50-60 \%, \times 10^{6}$

(d) $40-50 \%, \times 10^{5}$

(a)

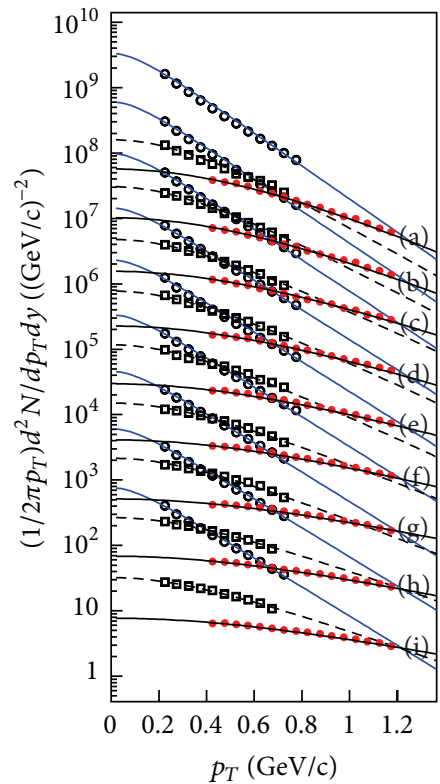

(e) $30-40 \%, \times 10^{4} \quad \circ \pi^{+}$

(f) $20-30 \%, \times 10^{3} \quad$ 口 $K^{+}$

(g) $10-20 \%, \times 10^{2}$

(h) $5-10 \%, \times 10^{1}$

(i) $0-50 \%, \times 10^{0}$

(b)

FIGURE 7: The same as for Figure 5, but showing the results at $\sqrt{s_{N N}}=200 \mathrm{GeV}$. 

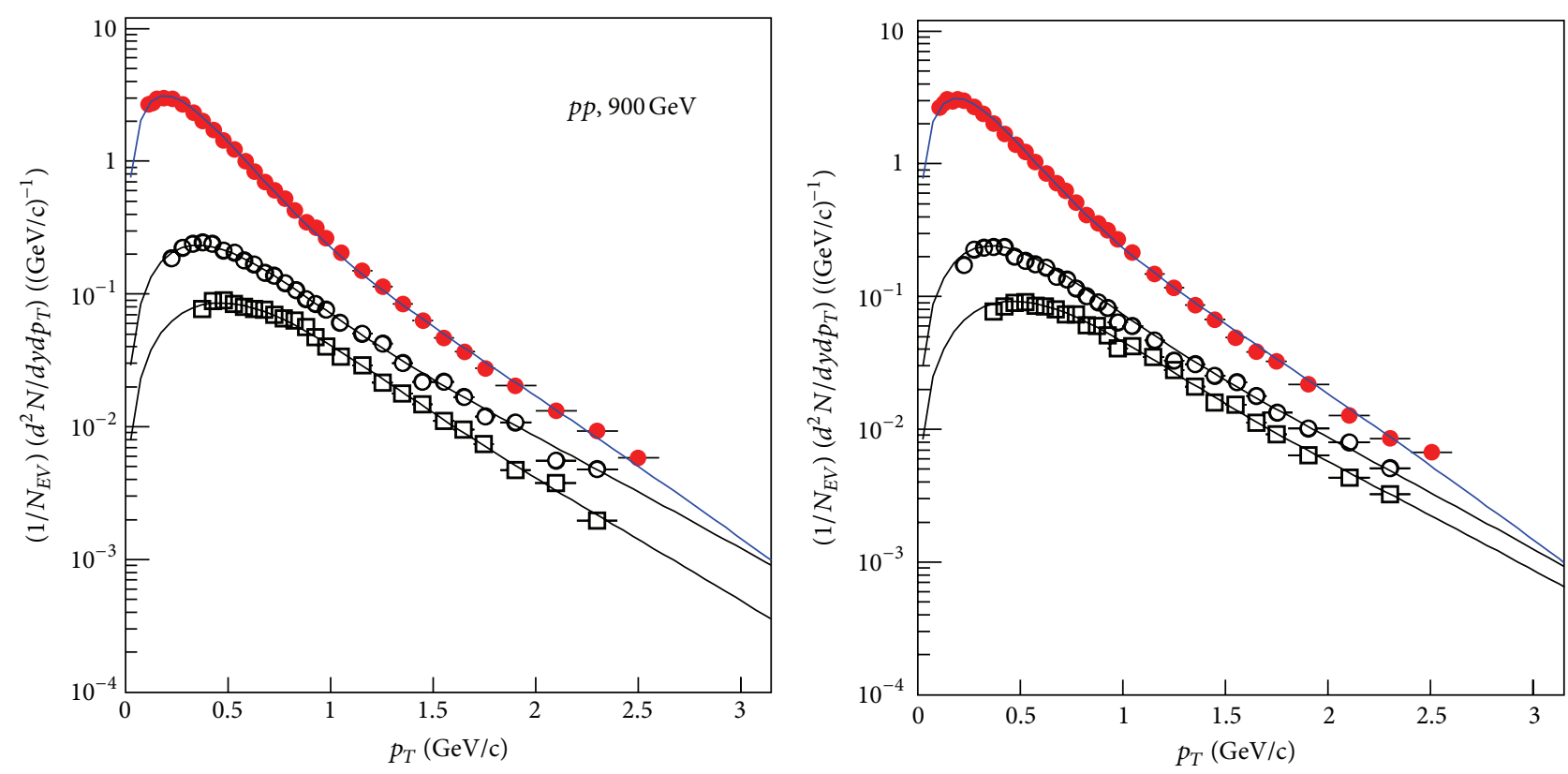

ㅇ $\pi^{-}$
- $\frac{K^{-}}{p}$

○ $\pi^{+}$

- $\mathrm{K}^{+}$

- $p$

(a)

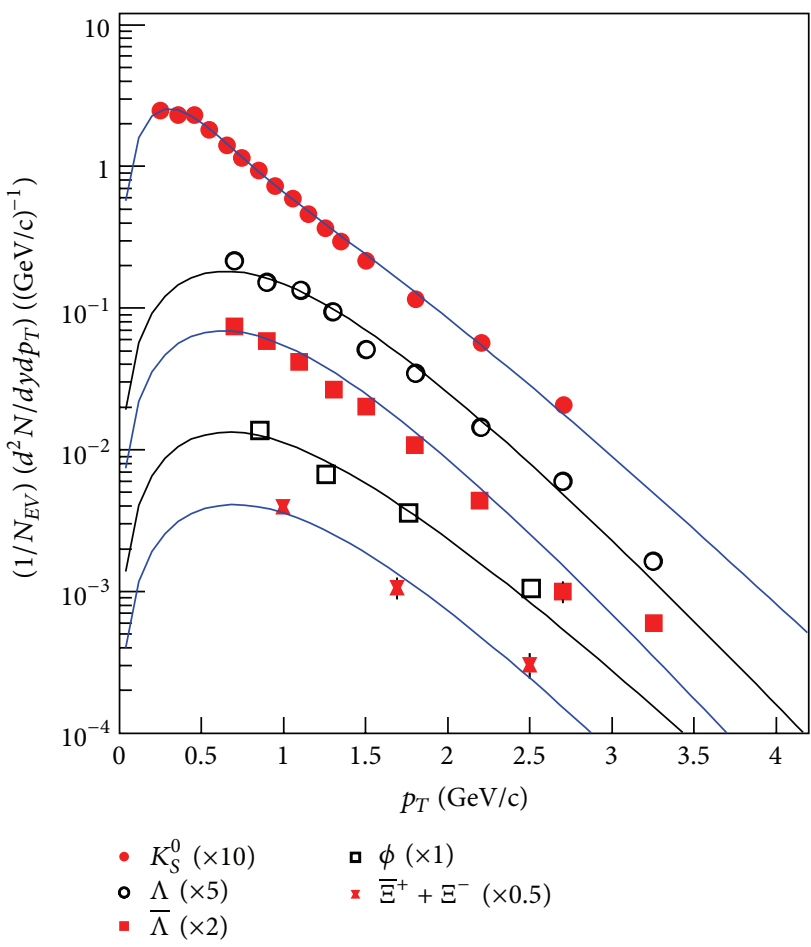

(c)

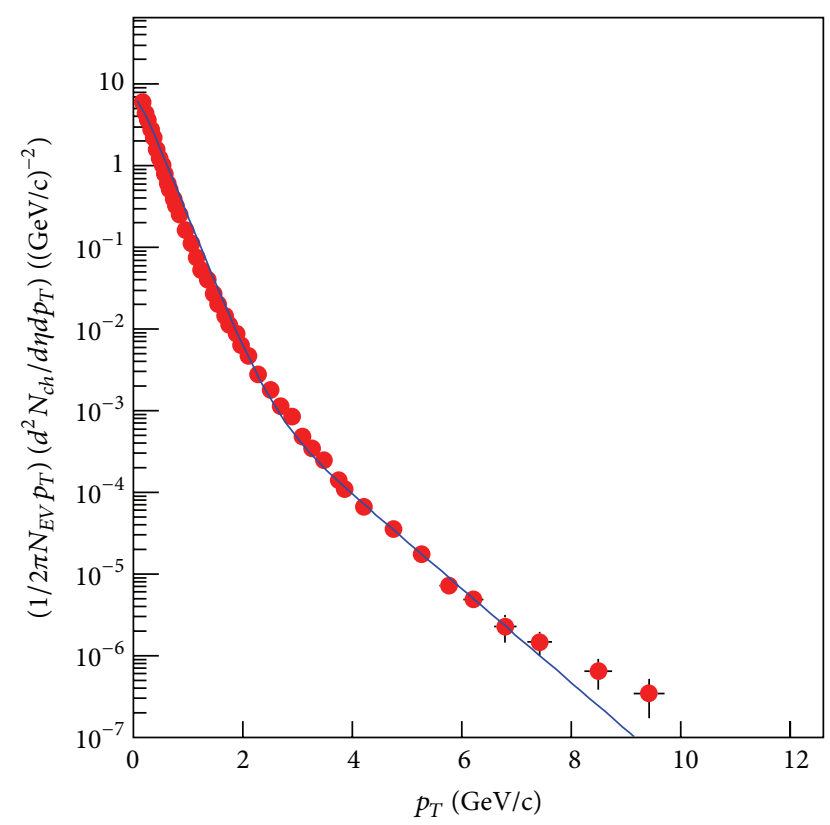

- NSD, charged particles, $|\eta|<0.8$

(d)

Figure 8: The transverse momentum distributions of (a) $\pi^{-}, K^{-}$, and $\bar{p}$, (b) $\pi^{+}, K^{+}$, and $p$, (c) $K_{S}^{0}, \Lambda, \bar{\Lambda}, \phi$, and $\bar{\Xi}^{+}+\Xi^{-}$, and (d) charged particles in range of $|\eta|<0.8$ in NSD produced in $p p$ collisions at $\sqrt{s}=900 \mathrm{GeV}$ with different magnifications are shown in the figure. The symbols represent the experimental data of the ALICE Collaboration $[33,34]$ and the curves are our results calculated by the Boltzmann or two-component Boltzmann distribution. 

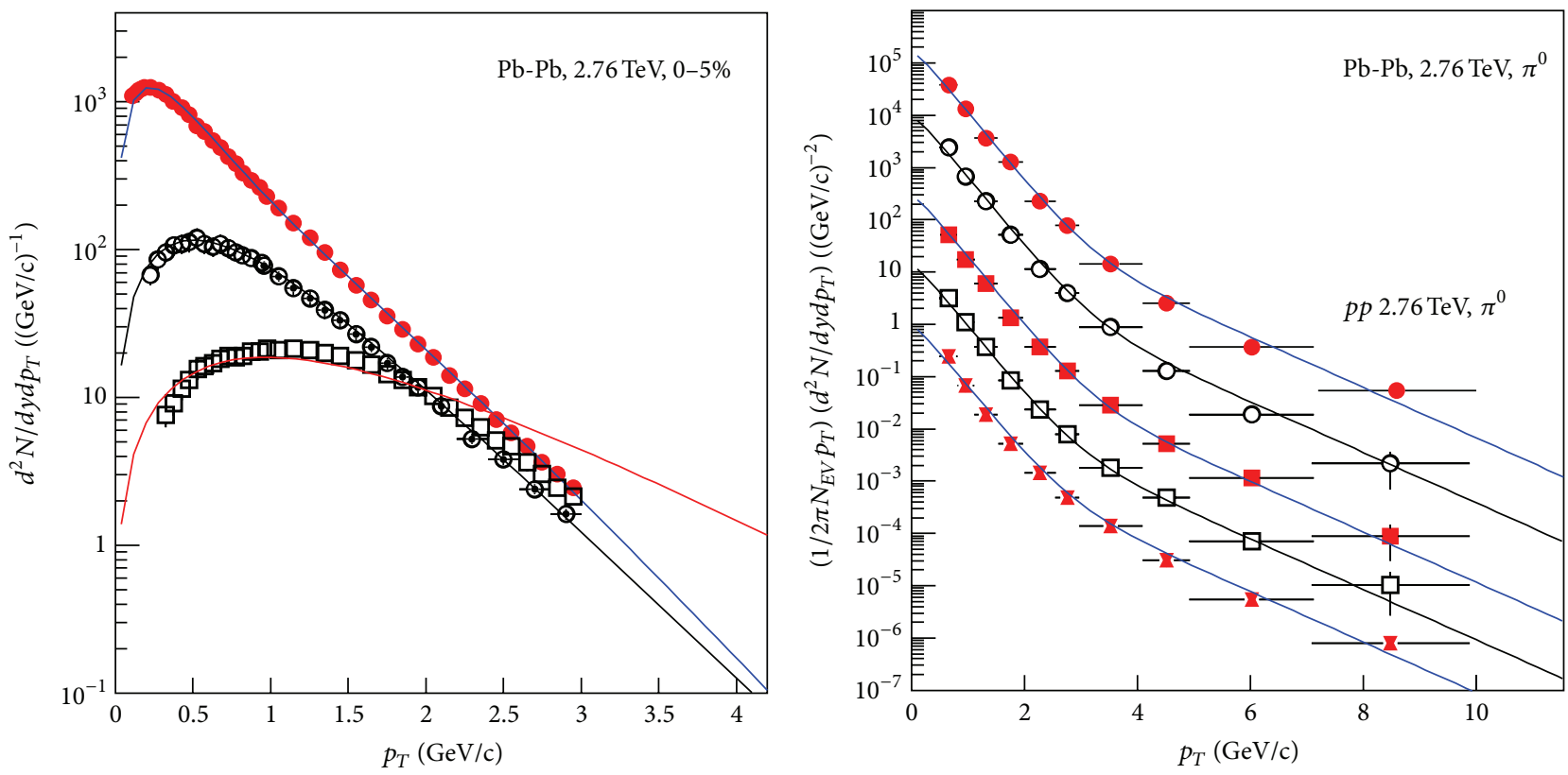

$\begin{array}{ll}\bullet \pi^{-} & \circ K^{-} \\ \circ K_{S}^{0} & \square \bar{p}\end{array}$
- $0-20 \%(\times 500)$
○ $20-40 \%(\times 50)$
- $40-60 \%(\times 4)$

(b)

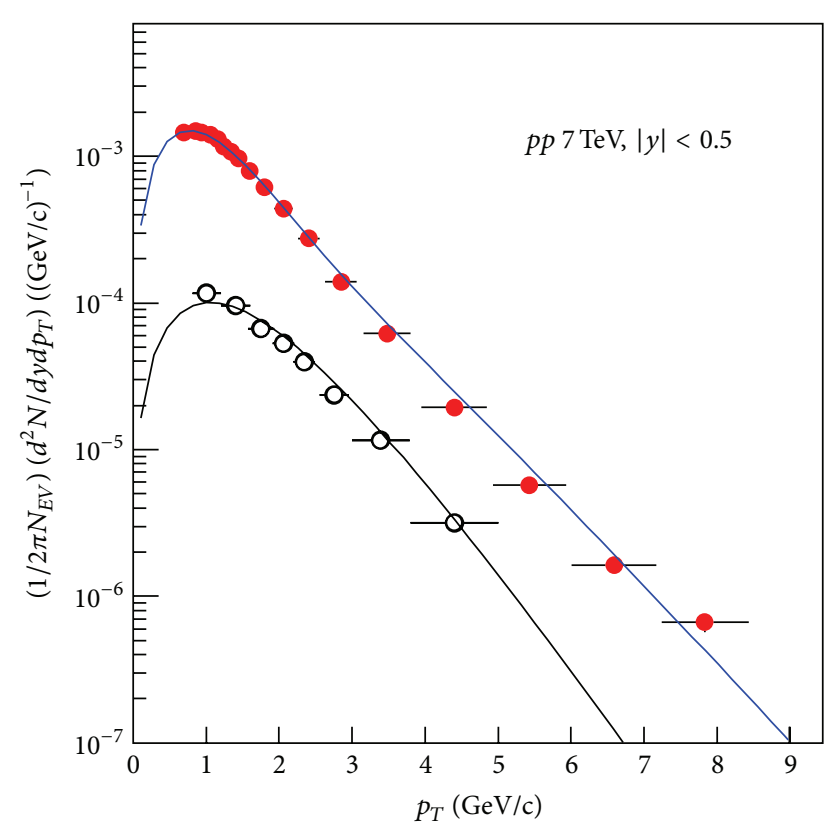

- $\Xi$

$\circ \Omega$
$5-10 \%(x 8)$

○ $5-10 \%(\times 8)$

- $10-20 \%(\times 5)$

ㅁ $20-30 \%(\times 3)$
$\mathrm{Pb}-\mathrm{Pb}, 2.76 \mathrm{TeV}, \bar{p},|y|<0.5$

$$
\begin{array}{ll}
\text { \ } & 40-50 \%(\times 1.5) \\
\text { จ } & 50-60 \%(\times 1) \\
\text { ○ } & 60-70 \%(\times 1)
\end{array}
$$$$
30-40 \%(\times 2)
$$

(c) (d)

Figure 9: The transverse momentum distributions of (a) $\pi^{-}, K_{S}^{0}+K^{-}$, and $\bar{p}$, (b) $\pi^{0}$, (c) $\bar{p}$, and (d) $\Xi$ and $\Omega$ produced in Pb-Pb collisions at $\sqrt{s_{N N}}=2.76 \mathrm{TeV}((\mathrm{a}),(\mathrm{b})$, and (c)) and $p p$ collision at $\sqrt{s}=2.76$ and $7 \mathrm{TeV}((\mathrm{b})$ and (d)) with different $y$ ranges, centrality classes, and magnifications are shown in the figure. The symbols represent the experimental data of the ALICE Collaboration [35-38] and the curves are our results calculated by the Boltzmann or two-component Boltzmann distribution. 
TABLE 8: Parameter values corresponding to the curves in Figure 8.

\begin{tabular}{|c|c|c|c|c|c|c|}
\hline Figure & Collision & Particle & $T_{1}(\mathrm{GeV})$ & $k_{1}$ & $T_{2}(\mathrm{GeV})$ & $\chi^{2} /$ dof \\
\hline \multirow{3}{*}{ Figure 8(a)/Figure 8(b) } & \multirow{3}{*}{$p p 900 \mathrm{GeV}$} & $\pi^{-} / \pi^{+}$ & $0.148 / 0.142$ & $0.803 / 0.765$ & $0.349 / 0.341$ & $0.153 / 0.369$ \\
\hline & & $K^{-} / K^{+}$ & 0.185 & 0.670 & 0.427 & $0.158 / 0.375$ \\
\hline & & $\bar{p} / p$ & 0.185 & $0.700 / 0.670$ & $0.390 / 0.427$ & $0.103 / 0.156$ \\
\hline \multirow{5}{*}{ Figure 8(c) } & \multirow{5}{*}{ pp $900 \mathrm{GeV}$} & $K_{S}^{0}$ & 0.132 & 0.505 & 0.369 & 0.375 \\
\hline & & $\Lambda$ & 0.323 & 1.000 & - & 1.026 \\
\hline & & $\bar{\Lambda}$ & 0.311 & 1.000 & - & 1.375 \\
\hline & & $\phi$ & 0.362 & 1.000 & - & 0.890 \\
\hline & & $\bar{\Xi}^{+}+\Xi^{-}$ & 0.325 & 1.000 & - & 0.664 \\
\hline Figure $8(\mathrm{~d})$ & $p p 900 \mathrm{GeV}$ & charged & 0.259 & 0.985 & 0.754 & 1.903 \\
\hline
\end{tabular}

radial flow and/or the early emission of heavy hadrons. The temperature does depend nonobviously on rapidity range (Figures 1(c)-1(e)).

Figure 2 shows the $p_{T}$ distributions of $J / \psi$ produced in (a) and (b) $p p$ collision at $\sqrt{s}=200 \mathrm{GeV}$, (c) $d$-Au collisions at $\sqrt{s_{N N}}=200 \mathrm{GeV}$, and (d) $p$-Pb collisions at beam energy being $400 \mathrm{GeV}$ with different $y$ ranges, global scale uncertainty (GSU) (or invariant mass $\left(M_{\mu^{+} \mu^{-}}\right)$) ranges, and magnifications shown in the figure, where $\sigma$ and $B$ denote the cross section and dilepton branching ratio, respectively. The symbols represent the experimental data of the PHENIX [28] (Figures 2(a) and 2(c)) [29, 30] (Figure 2(b)) and NA50 Collaborations [31] (Figure 2(d)), and the curves are our results calculated by the Boltzmann or two-component Boltzmann distributions. The values of parameters and $\chi^{2} /$ dof are given in Table 2 . We see again that the model with 1 or 2 sources describes the experimental data. For emission of $J / \psi$ the temperature parameter does depend nonobviously on rapidity range (Figures 2 (a) and $2(\mathrm{c})$ ).

The $p_{T}$ distributions of identified charged particles produced in $d$-Au collisions at $\sqrt{s_{N N}}=200 \mathrm{GeV}, \mathrm{Cu}-\mathrm{Cu}$ collisions at $\sqrt{s_{N N}}=22.5 \mathrm{GeV}$, Au-Au collisions at $\sqrt{s_{N N}}=$ $62.4,130$, and $200 \mathrm{GeV}$ with different centrality classes are displayed in Figures 3-7, respectively. The symbols represent the experimental data of the STAR [26] (Figures 3, 5, 6, and 7) and PHENIX Collaboration [32] (Figure 4), and the curves are our results calculated by the Boltzmann or twocomponent Boltzmann distributions. Correspondingly, the values of parameters and $\chi^{2} /$ dof are given in Tables 37 , respectively. Once more, the model with 1 or 2 sources describes the experimental data. From Tables 5, 6, and 7 we see clearly that for emissions of $\pi^{-}, K^{-}$, and $\bar{p}$ (Figures 5(a), 6(a), and 7(a)), as well as $\pi^{+}, K^{+}$, and $p$ (Figures 5(b), $6(\mathrm{~b})$, and $7(\mathrm{~b}))$, the temperature parameter increases with increases of particle mass, impact centrality, and $\sqrt{s_{N N}}$, where we would like to point out that a large centrality (a small percentage) corresponds to a small impact parameter. The similar conclusions can be obtained from Tables 3 and 4 .

Figure 8 gives the $p_{T}$ distributions of (a)-(c) identified particles and (d) charged particles in range of $|\eta|<0.8$ in nonsingle diffraction (NSD) produced in $p p$ collision at $\sqrt{s}=900 \mathrm{GeV}$, where $N_{\mathrm{EV}}$ and $N_{\mathrm{ch}}$ denote numbers of events and charged particles, respectively. The symbols represent the experimental data of the ALICE Collaboration [33, 34] and the curves are our results calculated by the Boltzmann or two-component Boltzmann distribution. The values of parameters and $\chi^{2} /$ dof are given in Table 8 . We see that the model with 1 or 2 sources describes the experimental data. For emissions of charged hadrons, the temperature parameter increases with increase of particle mass.

The $p_{T}$ distributions of identified particles produced in (a) central $\mathrm{Pb}-\mathrm{Pb}$ collisions at $\sqrt{s_{N N}}=2.76 \mathrm{TeV}$, (b) $\mathrm{Pb}$ $\mathrm{Pb}$ collisions with different centralities at $\sqrt{s_{N N}}=2.76 \mathrm{TeV}$ and inelastic $p p$ collision at $\sqrt{s}=2.76 \mathrm{TeV}$, (c) central rapidity region in $\mathrm{Pb}-\mathrm{Pb}$ collisions with different centralities at $\sqrt{s_{N N}}=2.76 \mathrm{TeV}$, and (d) central rapidity region in $p p$ collision at $\sqrt{s}=7 \mathrm{TeV}$ are presented in Figure 9. The symbols represent the experimental data of the ALICE Collaboration [35-38] and the curves are our results calculated by the Boltzmann or two-component Boltzmann distribution. The values of parameters and $\chi^{2} /$ dof are given in Table 9 . We see that in most cases the model with 1 or 2 sources describes the experimental data. Especially, for emissions of $\pi^{-}, K^{-}$, and $\bar{p}$ (Figure $9(\mathrm{a})$ ), the temperature parameter increases with increase of particle mass; for emission of $\pi^{0}$ (Figure 9(b)), the temperature parameter does not depend on impact centrality; and for emission of $\bar{p}$ (Figure 9(c)), the temperature parameter increases with increase of impact centrality (or with decrease of impact parameter).

From Tables 1-9 we see that some values of $\chi^{2} /$ dof are too low pointing to overestimated errors of the experimental points. In fact, in the case of errors being not available in related references, we have used a half size of the experimental points to give the errors. This treatment may cause larger errors in some cases.

\section{Discussions and Conclusions}

From the above discussions we see that the model used in the present work is just a simple phenomenology which does not contain other processes such as parton-hadron string dynamics, hydrodynamic flows, and resonances. The successful description renders that the mentioned processes should contribute a higher transverse momentum at multi$\mathrm{GeV}$ energy or a refined structure in distribution curve. In the concerned transverse momentum region and for the concerned distribution curves, we just need to consider the Maxwell-Boltzmann thermal law.

The present work is justified to compare fits in a low transverse momentum region $(<10 \mathrm{GeV} / \mathrm{c})$ for different particles 
TABLE 9: Parameter values corresponding to the curves in Figure 9.

\begin{tabular}{|c|c|c|c|c|c|c|c|}
\hline Figure & Collision & Particle & Type & $T_{1}(\mathrm{GeV})$ & $k_{1}$ & $T_{2}(\mathrm{GeV})$ & $\chi^{2} /$ dof \\
\hline \multirow{3}{*}{ Figure 9(a) } & \multirow{3}{*}{$\mathrm{Pb}-\mathrm{Pb} 2.76 \mathrm{TeV}$} & $\pi^{-}$ & $0-5 \%$ & 0.169 & 0.595 & 0.364 & 0.858 \\
\hline & & $K_{S}^{0}+K^{-}$ & $0-5 \%$ & 0.355 & 0.900 & 0.460 & 0.141 \\
\hline & & $\bar{p}$ & $0-5 \%$ & 0.697 & 1.000 & - & 0.832 \\
\hline \multirow{5}{*}{ Figure 9(b) } & \multirow{4}{*}{$\mathrm{Pb}-\mathrm{Pb} 2.76 \mathrm{TeV}$} & \multirow{5}{*}{$\pi^{0}$} & $0-20 \%$ & 0.332 & 0.985 & 0.900 & 1.321 \\
\hline & & & $20-40 \%$ & 0.332 & 0.985 & 0.900 & 1.760 \\
\hline & & & $40-60 \%$ & 0.332 & 0.985 & 0.900 & 1.324 \\
\hline & & & $60-80 \%$ & 0.332 & 0.975 & 0.900 & 0.583 \\
\hline & pp $2.76 \mathrm{TeV}$ & & Inelastic & 0.332 & 0.965 & 0.900 & 1.816 \\
\hline \multirow{8}{*}{ Figure 9(c) } & \multirow{8}{*}{$\mathrm{Pb}-\mathrm{Pb} 2.76 \mathrm{TeV}$} & \multirow{8}{*}{$\bar{p}$} & $0-5 \%,|y|<0.5$ & 0.632 & 1.000 & - & 0.517 \\
\hline & & & $5-10 \%,|y|<0.5$ & 0.600 & 1.000 & - & 0.324 \\
\hline & & & $10-20 \%,|y|<0.5$ & 0.580 & 1.000 & - & 0.275 \\
\hline & & & $20-30 \%,|y|<0.5$ & 0.540 & 1.000 & - & 0.200 \\
\hline & & & $30-40 \%,|y|<0.5$ & 0.532 & 1.000 & - & 0.275 \\
\hline & & & $40-50 \%,|y|<0.5$ & 0.461 & 1.000 & - & 0.432 \\
\hline & & & $50-60 \%,|y|<0.5$ & 0.450 & 1.000 & - & 0.296 \\
\hline & & & $60-70 \%,|y|<0.5$ & 0.385 & 1.000 & - & 0.371 \\
\hline \multirow{2}{*}{ Figure 9(d) } & \multirow{2}{*}{$p p 7 \mathrm{TeV}$} & $\Xi$ & $|y|<0.5$ & 0.350 & 0.700 & 0.730 & 0.510 \\
\hline & & $\Omega$ & $|y|<0.5$ & 0.565 & 1.000 & - & 0.666 \\
\hline
\end{tabular}

by the same thermal law. Although the difference for charged and neutral particles is unlikely due to Coulomb effects which are important for very soft charged particles only, both the charged and neutral particles obey the same thermal law. The transverse momentum can extend to more than $100 \mathrm{GeV} / \mathrm{c}$ at multi-GeV energy. The distribution in the low transverse momentum region is mainly contributed by the soft processes. The hard processes which contribute high transverse momentums can be partly described by the thermal law.

To conclude, we have used the multisource thermal model to describe the transverse momentum distributions of particles produced in the soft process in $p p$ and $A A$ collisions at RHIC and LHC energies. For single source, the relativistic ideal gas model is applied in description of particle behavior. The concerned distribution is finally described by single source or two sources which result from a Boltzmann or twocomponent Boltzmann distributions. The modeling results are in agreement with available experimental data, which renders that an equilibrium or two local equilibriums are reached in high energy collisions. Because of the evolvement time of interesting system in collisions being very short, the particles should reach rapidly to the state of equilibrium.

The present work can be used to extract nuclear temperature for soft process. For emissions of charged hadrons, the temperature parameter increases with increases of particle mass, impact centrality, and center-of-mass energy and does depend nonobviously on rapidity range. That the temperature increases with particle mass indicates the impact of radial flow and/or the early emission of heavy hadrons. The temperature parameter for emission of $J / \psi$ does depend nonobviously on rapidity range too, which is consistent with charged hadrons. However, for emission of $\pi^{0}$ the temperature parameter does not depend on impact centrality, which is inconsistent with charged hadrons. Different behaviors for $\pi^{0}$ and $\pi^{-} / \pi^{+}$render different production mechanisms.
Especially, there are Coulomb corrections for emissions of charged particles, which affects the extraction of temperature [39].

The values of temperature parameter for emissions of $\pi^{-} / \pi^{+}$are about $160-190 \mathrm{MeV}$ which reaches the temperature (166-172 MeV) of creating QGP at zero baryon-chemical potential, where $172 \mathrm{MeV}$ is the equilibrium phase transition temperature and $166 \mathrm{MeV}$ is due to finite hadron size [40]. In most cases the temperature for emission of heavy hadrons is greater than that for pions, which renders the impact of radial flow and/or the early emission of heavy hadrons in collisions.

\section{Conflict of Interests}

The authors declare that there is no conflict of interests regarding the publication of this paper.

\section{Acknowledgments}

This work was supported by the National Natural Science Foundation of China under Grant no. 10975095 and no. 11247250, the China National Fundamental Fund of Personnel Training under Grant no. J1103210, the Open Research Subject of the Chinese Academy of Sciences Large-Scale Scientific Facility under Grant no. 2060205, and the Shanxi Scholarship Council of China.

\section{References}

[1] B. B. Back, M. D. Baker, D. S. Barton et al., "Charged-particle pseudorapidity density distributions from $\mathrm{Au}+\mathrm{Au}$ collisions at $\sqrt{s_{N N}}=130 \mathrm{GeV}$,' Physical Review Letters, vol. 87, no. 10, Article ID 102303, 2001.

[2] I. G. Bearden, D. Beavis, C. Besliu et al., "Pseudorapidity distributions of charged particles from $\mathrm{Au}+\mathrm{Au}$ collisions at the 
maximum RHIC energy, $\sqrt{s_{N N}}=200 \mathrm{GeV}$,' Physical Review Letters, vol. 88, no. 20, Article ID 202301, 2001.

[3] A. Toia and ALICE Collaboration, "Bulk properties of $\mathrm{Pb}-\mathrm{Pb}$ collisions at $\sqrt{s_{N N}}=2.76 \mathrm{TeV}$ measured by ALICE," Journal of Physics G, vol. 38, no. 12, Article ID 124007, 2011.

[4] H. Song, S. A. Bass, U. Heinz, T. Hirano, and C. Shen, "200 $\mathrm{A} \mathrm{GeV} \mathrm{Au+Au} \mathrm{collisions} \mathrm{serve} \mathrm{a} \mathrm{nearly} \mathrm{perfect} \mathrm{quark-gluon}$ liquid," Physical Review Letters, vol. 106, no. 19, Article ID 192301, 2011.

[5] G. Roland, "QCD studies with CMS at LHC", in INT Program 10-2a Quantifying the Properties of Hot QCD Matter, Seattle, Wash, USA, May 2010.

[6] F. Gelis, "Initial stages of heavy-ion collisions from the CGC," in INT Program 10-2a Quantifying the Properties of Hot QCD Matter, Seattle, Wash, USA, May 2010.

[7] A. Dumitru, "CGC in heavy-ion coll," in INT Program 10-2a Quantifying the Properties of Hot QCD Matter, Seattle, Wash, USA, May 2010.

[8] B. Alver, B. B. Back, M. D. Baker et al., "Charged-particle multiplicity and pseudorapidity distributions measured with the PHOBOS detector in $\mathrm{Au}+\mathrm{Au}, \mathrm{Cu}+\mathrm{Cu}, \mathrm{d}+\mathrm{Au}$, and $\mathrm{p}+\mathrm{p}$ collisions at ultrarelativistic energies ," Physical Review C, vol. 83, no. 2, Article ID 024913, 2011.

[9] F. Abe, D. Amidei, G. Apollinari et al., "Pseudorapidity distributions of charged particles produced in $\bar{p} p$ interactions as $\sqrt{s}=630$ and 1800 GeV," Physical Review D, vol. 41, no. 7, pp. 2330-2333, 1990.

[10] I. G. Bearden, D. Beavis, C. Besliu et al., "Nuclear stopping in $\mathrm{Au}+\mathrm{Au}$ collisions at $\sqrt{{s_{N N}}}=200 \mathrm{GeV}$," Physical Review Letters, vol. 93, no. 10, Article ID 102301, 2004.

[11] C. Alt, T. Anticic, B. Baatar et al., "Energy dependence of $\phi$ meson production in central $\mathrm{Pb}+\mathrm{Pb}$ collisions at $\sqrt{s_{N N}}=6$ to 17 GeV," Physical Review C, vol. 78, no. 4, Article ID 044907, 2008.

[12] S. Chatrchyan, V. Khachatryan, A. M. Sirunyan et al., "Missing transverse energy performance of the CMS detector," Journal of Instrumentation, vol. 6, no. 9, Article ID P09001, 2011.

[13] The CMS Collaboration, "Missing transverse energy performance in minimum-bias and jet events from proton-proton collisions at $\sqrt{s}=7$," Preprint CMS-PAS-JME-10-004, 2010.

[14] G. Aad, B. Abbott, J. Abdallah et al., "Measurement of the jet fragmentation function and transverse profile in proton-proton collisions at a center-of-mass energy of $7 \mathrm{TeV}$ with the ATLAS detector," European Physical Journal C, vol. 71, no. 11, article 1795, 2011.

[15] J. Adams, M. M. Aggarwal, Z. Ahammed et al., "K(892)* resonance production in $\mathrm{Au}+\mathrm{Au}$ and $\mathrm{p}+\mathrm{p}$ collisions at $\sqrt{\boldsymbol{s}_{N N}}=$ 200 GeV," Physical Review C, vol. 71, no. 6, Article ID 064902, 2005.

[16] S. Abreu, N. Borghini, S. Jeon et al., "Heavy-ion collisions at the LHC-last call for predictions," Journal of Physics G, vol. 35, no. 5, Article ID 054001, 2008.

[17] K. Urmossy, "Multiplicity dependence of hadron spectra in proton-proton collisions at LHC energies and super-statistics," http://arxiv.org/abs/1212.0260 .

[18] H. R. Wei, Y. H. Chen, L. N. Gao, and F. H. Liu, "On multi-component Erlang distribution and Levy distribution of transverse momentum spectra in high-energy collisions ," submitted to. Advances in High Energy Physics.

[19] F. H. Liu, "Unified description of multiplicity distributions of final-state particles produced in collisions at high energies," Nuclear Physics A, vol. 810, no. 1-4, pp. 159-172, 2008.
[20] F. H. Liu and J. S. Li, "Isotopic production cross section of fragments in ${ }^{56} \mathrm{Fe}+\mathrm{p}$ and ${ }^{136} \mathrm{Xe}\left({ }^{124} \mathrm{Xe}\right)+\mathrm{Pb}$ reactions over an energy range from 300A to $1500 \mathrm{~A} \mathrm{MeV,"} \mathrm{Physical} \mathrm{Review} \mathrm{C,} \mathrm{vol.}$ 78, no. 4, Article ID 044602, 2008.

[21] P. Z. Ning, L. Li, and D. F. Min, Foundation of Nuclear Physics: Nucleons and Nuclei, Higher Education Press, Beijing, China, 2003.

[22] F. H. Liu, C. X. Tian, M. Y. Duan, and B. C. Li, "Relativistic and quantum revisions of the multisource thermal model in highenergy collisions," Advances in High Energy Physics, vol. 2012, Article ID 287521, 9 pages, 2012.

[23] C. D. Dermer, "The production spectrum of a relativistic Maxwell-Boltzmann gas," The Astrophysical Journal, vol. 280, no. 1, pp. 328-333, 1984.

[24] J. L. Synge, The Relativistic Gas, North-Holland, Amsterdam, The Netherlands, 1957.

[25] C. R. Meng, "Transverse momentum and rapidity distributions of $\phi$ Mesons produced in $\mathrm{Pb}-\mathrm{Pb}$ collisions at SPS energies," Chinese Physics Letters, vol. 26, no. 10, Article ID 102501, 2009.

[26] B. I. Abelev, M. M. Aggarwal, Z. Ahammed et al., "Systematic measurements of identified particle spectra in pp, $d+A u$, and $\mathrm{Au}+\mathrm{Au}$ collisions at the STAR detector," Physical Review C, vol. 79, no. 3, Article ID 034909, 2009.

[27] H. Yang and BRAHMS Collaboration, "Identified particle production in $\mathrm{p}+\mathrm{p}$ and $\mathrm{d}+\mathrm{Au}$ collisions at RHIC," Journal of Physics G, vol. 34, no. 8, pp. S619-S622, 2007.

[28] A. Adare, S. S. Adler, S. Afanasiev et al., "Cold nuclear matter effects on $J / \psi$ production as constrained by deuteron-gold measurements at $\sqrt{s_{N N}}=200 \mathrm{GeV}$, Physical Review C, vol. 77, no. 2, Article ID 024912, 2008.

[29] A. Adare, S. Afanasiev, C. Aidala et al., "J/ $\psi$ Production versus transverse momentum and rapidity in $\mathrm{p}+\mathrm{p}$ collisions at $\sqrt{s_{N N}}=$ 200 GeV," Physical Review Letters, vol. 98, no. 23, Article ID 232002, 2007.

[30] A. Bickley and PHENIX Collaboration, "Heavy quarkonia production in $\mathrm{p}+\mathrm{p}$ collisions from the PHENIX experiment," Journal of Physics G, vol. 34, no. 8, pp. S779-S782, 2007.

[31] B. Alessandro, C. Alexa, R. Arnaldi et al., " $J / \psi$ and $\psi$ ' production and their normal nuclear absorption in proton-nucleus collisions at $400 \mathrm{GeV}$," European Physical Journal C, vol. 48, no. 2, pp. 329-341, 2006.

[32] J. T. Mitchell and PHENIX Collaboration, "The PHENIX potential in the search for the QCD critical point," in Proceedings of the 3rd International Workshop on the Critical Point and Onset of Deconfinement, Florence, Italy, July 2006, http://arxiv .org/abs/nucl-ex/0701079.

[33] M. Kowalski and ALICE Collaboration, "First results on charged particle production in ALICE experiment at LHC," Acta Physica Polonica B, vol. 42, no. 3-4, pp. 859-866, 2011.

[34] K. Aamodt, A. Abrahantes Quintana, D. Adamova' et al., "Strange particle production in proton-proton collisions at $\sqrt{s}=$ $0.9 \mathrm{TeV}$ with ALICE at the LHC," European Physical Journal C, vol. 71, no. 3, Article ID 1594, 2011.

[35] M. van Leeuwen and ALICE Collaboration, "High- $p_{T}$ results from ALICE," in Proceedings of the Hadron Collider Physics Symposium, Paris, France, November 2012, http://arxiv.org/abs/ 1201.5205 .

[36] M. Floris and ALICE Collaboration, "Identified particles in pp and $\mathrm{Pb}-\mathrm{Pb}$ collisions at LHC energies with the ALICE detector," Journal of Physics G, vol. 38, no. 12, Article ID 124025, 2011. 
[37] H. Appelshäuser and ALICE Collaboration, "Particle production at large transverse momentum with ALICE," Journal of Physics G, vol. 38, no. 12, Article ID 124014, 2011.

[38] R. Preghenella and ALICE Collaboration, "Transverse momentum spectra of identified charged hadrons with the ALICE detector in $\mathrm{Pb}-\mathrm{Pb}$ collisions at $\sqrt{s_{N N}}=2.76 \mathrm{TeV}$,' in Proceedings of the Europhysics Conference on High Energy Physics, Rhône-Alpes, France, July 2011, http://arxiv.org/abs/1111.0763.

[39] H. Zheng, G. Giuliani, and A. Bonasera, "Coulomb corrections to density and temperature of bosons in heavy ioncollisions," http://arxiv-web3.library.cornell.edu/abs/1306.5741.

[40] J. Letessier, G. Torrieri, S. Hamieh, and J. Rafelski, "Quark-gluon plasma fireball explosion," Journal of Physics G, vol. 27, no. 3, pp. 427-437, 2001. 

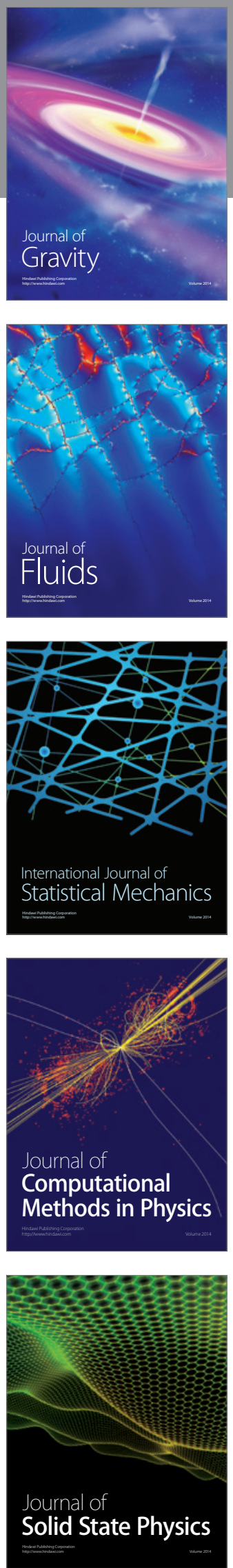

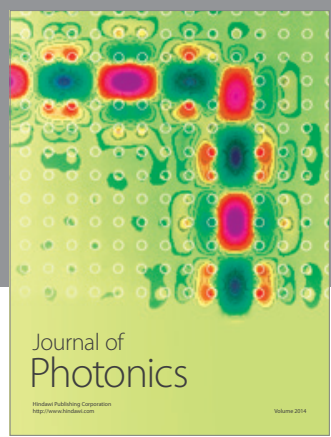

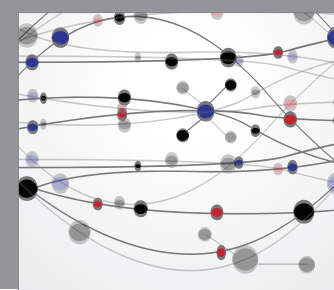

The Scientific World Journal

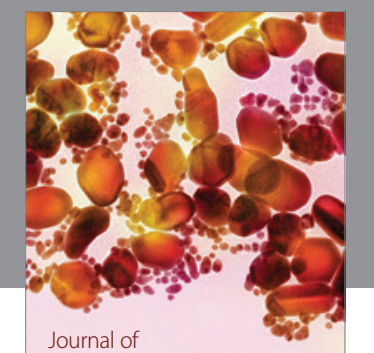

Soft Matter
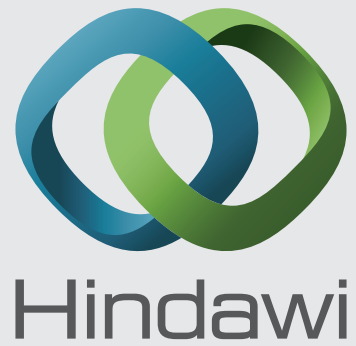

Submit your manuscripts at

http://www.hindawi.com
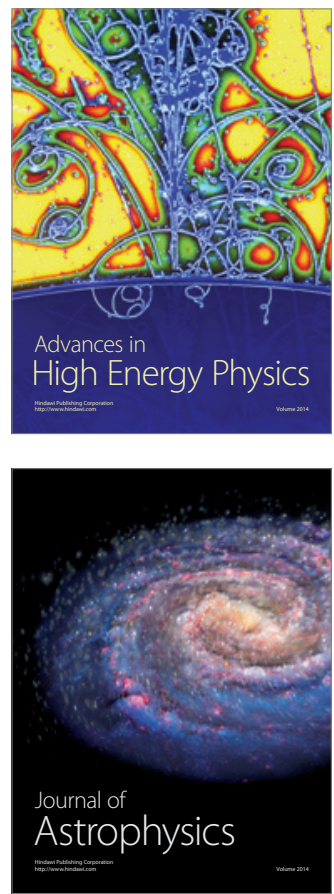
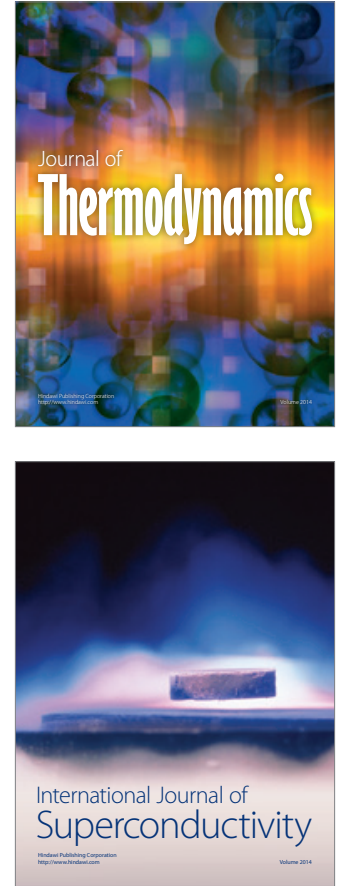
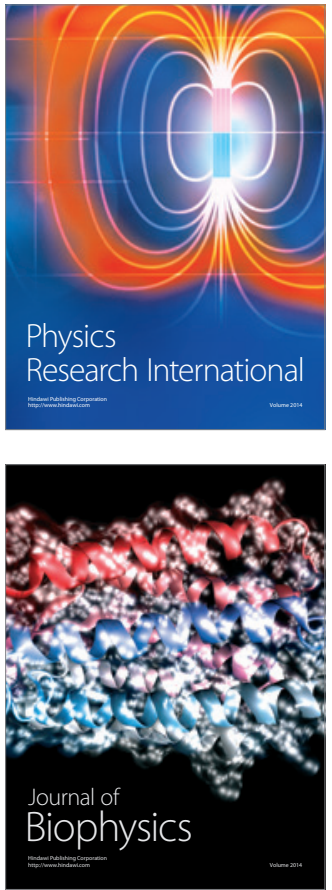
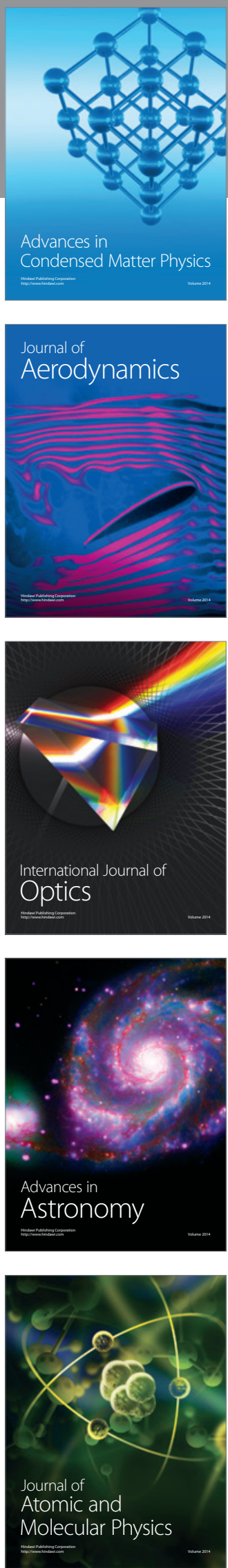